\title{
Sobolev-Type Spaces on the Dual of the Chébli-Trimèche Hypergroup and Applications
}

\author{
Mourad Jelassi ${ }^{1}$ and Hatem Mejjaoli ${ }^{2}$ \\ ${ }^{1}$ Department of Mathematics, ISSAT Mateur, Carthage University, Bizerte, 7030 Mateur, Tunisia \\ ${ }^{2}$ Department of Mathematics, College of Sciences, Taibah University, P.O. Box 30002, Al Madinah Al Munawarah, Saudi Arabia
}

Correspondence should be addressed to Mourad Jelassi; mourad.jelassi@fst.rnu.tn

Received 4 December 2013; Accepted 30 December 2013; Published 4 March 2014

Academic Editor: S. A. Mohiuddine

Copyright (c) 2014 M. Jelassi and H. Mejjaoli. This is an open access article distributed under the Creative Commons Attribution License, which permits unrestricted use, distribution, and reproduction in any medium, provided the original work is properly cited.

\begin{abstract}
We define and study Sobolev-type spaces $W_{A}^{s, p}\left(\mathbb{R}_{+}\right)$associated with singular second-order differential operator on $(0, \infty)$. Some properties are given; in particular we establish a compactness-type imbedding result which allows a Reillich-type theorem. Next, we introduce a generalized Weierstrass transform and, using the theory of reproducing kernels, some applications are given.
\end{abstract}

\section{Introduction}

The Sobolev spaces have served as a very useful tool in the theory of partial differential equations, mostly those related to continuum mechanics or physics. Their uses and the study of their properties were facilitated by the theory of distributions and Fourier analysis. The Sobolev space $W^{s, p}\left(\mathbb{R}_{+}\right)$is defined by the use of the classical Fourier transform as the set of all tempered distribution $u$ such that its classical Fourier transform $\widehat{u}$ satisfying

$$
\left(1+|\xi|^{2}\right)^{s / 2} \widehat{u} \in L^{p}\left(\mathbb{R}_{+}\right) .
$$

Generalization of the Sobolev space has been studied by replacing the classical Fourier transform by a generalized one.

In this paper we consider the differential operator on $(0, \infty)$,

$$
\Delta_{A}=\frac{d^{2}}{d x^{2}}+\frac{A^{\prime}(x)}{A(x)} \frac{d}{d x}+\rho^{2}, \quad \rho>0,
$$

where $A$ is the Chebli-Trimeche function (cf. [1, Section 3.5]) defined on $[0, \infty)$ and satisfies the following conditions.

(i) There exists a positive even infinitely differentiable function $B$ on $\mathbb{R}$, with $B(x) \geq 1, x \in \mathbb{R}_{+}$, such that $A(x)=x^{2 \alpha+1} B(x), \alpha>-1 / 2$. (ii) $A$ is increasing on $\mathbb{R}_{+}$and $\lim _{x \rightarrow \infty} A(x)=\infty$.

(iii) $A^{\prime} / A$ is decreasing on $(0, \infty)$, and $\lim _{x \rightarrow \infty}\left(A^{\prime}(x) / A(x)\right)=2 \rho$.

(iv) There exists a constant $\sigma>0$, such that for all $x \in$ $\left[x_{0}, \infty\right), x_{0}>0$, we have

$$
\frac{A^{\prime}(x)}{A(x)}= \begin{cases}2 \rho+e^{-\sigma x} F(x), & \text { if } \rho>0 \\ \frac{2 \alpha+1}{x}+e^{-\sigma x} F(x), & \text { if } \rho=0,\end{cases}
$$

where $F$ is $C^{\infty}$ on $(0, \infty)$, bounded together with its derivatives.

For $A(x)=x^{2 \alpha+1}, \alpha>-1 / 2$, and $\rho=0$, we regain the Bessel operator

$$
l_{\alpha} f=\frac{d^{2} f}{d x^{2}}+\left(\frac{2 \alpha+1}{x}\right) \frac{d f}{d x}
$$

For $A(x)=\sinh ^{2 \alpha+1}(x) \cosh ^{2 \beta+1}(x), \alpha \geq \beta \geq-1 / 2, \alpha \neq-$ $1 / 2$, and $\rho=\alpha+\beta+1$, we regain the Jacobi operator

$$
\begin{aligned}
l_{\alpha, \beta} f= & \frac{d^{2} f}{d x^{2}}+[(2 \alpha+1) \operatorname{coth} x+(2 \beta+1) \tanh x] \\
& \times \frac{d f}{x}+\rho^{2} .
\end{aligned}
$$


The purpose of this paper is to introduce and study new Sobolev-type spaces $W_{A}^{s, p}\left(\mathbb{R}_{+}\right)$, associated with the singular operator $\Delta_{A}$ that generalizes the corresponding classical spaces. The Bessel case was treated by Assal and Nessibi [2], while Ben Salem and Dachraoui [3] studied the generalized Soblev spaces in the Jacobi setting theory.

The paper is organized as follows. In Section 2 we recall the main results about the harmonic analysis associated with the operator $\Delta_{A}$. In Section 3 Sobolev-type spaces on the dual of the Chébli-Trimèche hypergroup are studied. Some properties including completeness and Sobolev embedding theorems are established. Next, we prove a Reillich-type theorem. Finally, in Section 4, as applications, we give practical real inversion formulas using the theory of reproducing kernels for the generalized Weierstrass transform.

\section{Preliminaries}

In this section, we collect some harmonic analysis results related to the operator $\Delta_{A}$. For details, we refer the reader to $[1,4-8]$.

2.1. Eigenfunctions of the Operator $\Delta_{A}$. In the following, we denote by

$\mathscr{E}_{*}(\mathbb{R})$ the space of even $C^{\infty}$-functions on $\mathbb{R}$,

$\mathcal{S}_{*}(\mathbb{R})$ the subspace of $\mathscr{E}_{*}(\mathbb{R})$, consisting of functions $f$ rapidly decreasing together with their derivatives,

$\mathcal{S}_{*}^{2}(\mathbb{R})=\varphi_{0} \mathcal{S}_{*}(\mathbb{R})$, where $\varphi_{0}$ is the eigenfunction of the operator $\Delta_{A}$ associated with the value $\lambda=0$,

$\delta_{*}^{\prime}(\mathbb{R})$ the dual topological space of $\delta_{*}(\mathbb{R})$,

$\left(\mathcal{S}_{*}^{2}\right)^{\prime}(\mathbb{R})$ the dual topological space of $\mathcal{S}_{*}^{2}(\mathbb{R})$,

$\mathscr{E}_{*}^{\prime}\left(\mathbb{R}_{+}\right)$the dual topological space of $\mathscr{E}_{*}(\mathbb{R})$,

$\mathscr{H}_{*}(\mathbb{C})$ the space of even entire functions on $\mathbb{C}$ which are of exponential type and slowly increasing,

$\mathscr{H}_{*, a}(\mathbb{C})$ the subspace of $\mathscr{H}_{*}(\mathbb{C})$ satisfying

$\exists m \in \mathbb{N}$.

$$
P_{m}(f)=\sup _{\lambda \in \mathbb{C}}\left(1+\lambda^{2}\right)^{-m}|f(\lambda)| \exp (-a|\operatorname{Im} \lambda|)<+\infty .
$$

We have $\mathscr{H}_{*}(\mathbb{C})=\bigcup_{a \geq 0} \mathscr{H}_{*, a}(\mathbb{C})$

For every $\lambda \in \mathbb{C}$, let us denote by $\varphi_{\lambda}$ the unique solution of the eigenvalue problem:

$$
\begin{gathered}
\Delta_{A} f(x)=-\lambda^{2} f(x), \\
f(0)=1, \quad f^{\prime}(0)=0 .
\end{gathered}
$$

Remark 1. This function satisfies the following properties.

(i) $\forall x \geqslant 0$, the function $\lambda \mapsto \varphi_{\lambda}(x)$ is analytic on $\mathbb{C}$.

(ii) Product formula:

$$
\begin{gathered}
\forall x, y \geqslant 0 \\
\varphi_{\lambda}(x) \varphi_{\lambda}(y)=\int_{0}^{\infty} \varphi_{\lambda}(z) w(x, y, z) A(z) d z
\end{gathered}
$$

where $w(x, y, \cdot)$ is a measurable positive function on $[0, \infty)$, with support in $[|x-y|, x+y]$.

(iii)

$$
\forall \lambda \geq 0, \quad x \in \mathbb{R}, \quad\left|\varphi_{\lambda}(x)\right| \leq 1
$$

(iv) For $\rho>0$, we have

$$
\begin{gathered}
\forall x \geq 0, \quad \forall \lambda \in \mathbb{R}, \\
\left|\varphi_{\lambda}(x)\right| \leq \varphi_{0}(x) \leq m(1+x) \exp (-\rho x),
\end{gathered}
$$

where $m$ is a positive constant.

(v) For $\rho=0$, we have

$$
\forall x \geq 0, \quad \varphi_{0}(x)=1 .
$$

(vi) We have the following integral representation of Mehler type,

$$
\forall x>0, \quad \forall \lambda \in \mathbb{C}, \quad \varphi_{\lambda}(x)=\int_{0}^{x} k(x, t) \cos (\lambda t) d t,
$$

where $k(x, \cdot)$ is an even positive $C^{\infty}$ function on $(-x, x)$ with support in $[-x, x]$.

2.2. Generalized Fourier Transform. For a Borel positive measure $\mu$ on $\mathbb{R}$, and $1 \leq p \leq \infty$, we write $L_{\mu}^{p}\left(\mathbb{R}_{+}\right)$for the Lebesgue space equipped with the norm $\|\cdot\|_{L_{\mu}^{p}\left(\mathbb{R}_{+}\right)}$defined by

$$
\|f\|_{L_{\mu}^{p}\left(\mathbb{R}_{+}\right)}=\left(\int_{\mathbb{R}}|f(x)|^{p} d \mu(x)\right)^{1 / p}, \quad \text { if } p<\infty,
$$

and $\|f\|_{L_{\mu}^{\infty}\left(\mathbb{R}_{+}\right)}=\operatorname{ess~sup}_{x \in \mathbb{R}_{+}}|f(x)|$. When $\mu(x)=w(x) d x$, with $w$ a nonnegative function on $\mathbb{R}_{+}$, we replace the $\mu$ in the norms by $w$.

For $f \in L_{A}^{1}\left(\mathbb{R}_{+}\right)$, the generalized Fourier transform is defined by

$$
\mathscr{F}(f)(\lambda)=\int_{\mathbb{R}_{+}} f(x) \varphi_{\lambda}(x) A(x) d x, \quad \forall \lambda \in \mathbb{R} .
$$

The inverse generalized Fourier transform of a suitable function $g$ on $\mathbb{R}_{+}$is given by

$$
\mathscr{J} g(x)=\mathscr{F}^{-1} g(x)=\int_{\mathbb{R}_{+}}(\lambda) \varphi_{\lambda}(x) d \gamma(\lambda),
$$

where $d \gamma(\lambda)$ is the spectral measure given by

$$
d \gamma(\lambda)=\frac{d \lambda}{\left|c_{A}(\lambda)\right|^{2}}
$$

Remark 2. The function $\lambda \mapsto c_{A}(\lambda)$ satisfies the following properties.

(i) For $\lambda \in \mathbb{R}$, we have $c_{A}(-\lambda)=\overline{c_{A}(\lambda)}$.

(ii) The function $\left|c_{A}(\lambda)\right|^{-2}$ is continuous on $[0, \infty[$. 
(iii) There exist positive constants $k_{1}, k_{2}$, and $k_{3}$, such that If $\rho \geqslant 0: \forall \lambda \in \mathbb{C}, \operatorname{Im} \lambda \leqslant 0,|\lambda|>k_{3}$;

$$
k_{1}|\lambda|^{2 \alpha+1} \leqslant\left|c_{A}(\lambda)\right|^{-2} \leqslant k_{2}|\lambda|^{2 \alpha+1} .
$$

If $\rho=0, \alpha>0: \forall \lambda \in \mathbb{C},|\lambda| \leqslant k_{3}$;

$$
k_{1}|\lambda|^{2 \alpha+1} \leqslant\left|c_{A}(\lambda)\right|^{-2} \leqslant k_{2}|\lambda|^{2 \alpha+1} .
$$

If $\rho>0: \forall \lambda \in \mathbb{C},|\lambda| \leqslant k_{3}$;

$$
k_{1}|\lambda|^{2} \leqslant\left|c_{A}(\lambda)\right|^{-2} \leqslant k_{2}|\lambda|^{2} .
$$

Proposition 3 (see $[7,9]$ ). (i) The generalized transform $\mathscr{F}$ and its inverse $\mathcal{F}$ are topological isomorphisms between the generalized Schwartz space $\mathcal{S}_{*}^{2}(\mathbb{R})$ and the Schwartz space $\mathcal{S}\left(\mathbb{R}_{*}\right)$.

(ii) The transform $\mathscr{F}$ is a topological isomorphism from $\mathscr{E}_{*}^{\prime}\left(\mathbb{R}_{+}\right)$onto $\mathscr{H}_{*}(\mathbb{C})$. Moreover, for all $T \in \mathscr{E}_{*}^{\prime}\left(\mathbb{R}_{+}\right)$, we have $\operatorname{supp}(T) \subseteq[-a, a]$ if and only if $\mathscr{F}(T) \in \mathscr{H}_{*, a}(\mathbb{C})$.

Next, we give some properties of this transform.

(i) For $f$ in $L_{A}^{1}\left(\mathbb{R}_{+}\right)$we have

$$
\|\mathscr{F}(f)\|_{L_{\gamma}^{\infty}\left(\mathbb{R}_{+}\right)} \leq\|f\|_{L_{A}^{1}\left(\mathbb{R}_{+}\right)} .
$$

(ii) For $f$ in $\mathcal{S}_{*}^{2}(\mathbb{R})$ we have

$$
\mathscr{F}\left(\Delta_{A} f\right)(y)=-y^{2} \mathscr{F}(f)(y), \quad \forall y \in \mathbb{R}_{+} .
$$

Proposition 4 (see $[7,9])$. Plancherel formula for $\mathscr{F}$. For all $f$ in $\delta_{*}^{2}(\mathbb{R})$, we have

$$
\int_{\mathbb{R}_{+}}|f(x)|^{2} A(x) d x=\int_{\mathbb{R}_{+}}|\mathscr{F}(f)(\xi)|^{2} d \gamma(\xi) .
$$

(ii) Plancherel Theorem. The transform $\mathscr{F}$ extends uniquely to an isomorphism from $L_{A}^{2}\left(\mathbb{R}_{+}\right)$onto $L_{\gamma}^{2}\left(\mathbb{R}_{+}\right)$.

Remark 5. We have $\mathcal{S}_{*}^{2}(\mathbb{R}) \subset L_{A}^{p}\left(\mathbb{R}_{+}\right)$for all $2 \leq p \leq \infty$, but $S_{*}^{2}(\mathbb{R}) \nsubseteq L_{A}^{p}\left(\mathbb{R}_{+}\right)$for all $0<p<2$.

Proposition 6. Let $1 \leq p \leq 2$. The Fourier transform $\mathscr{F}$ (resp. J) can be extended as a continuous mapping from $L_{A}^{p}\left(\mathbb{R}_{+}\right)$ onto $L_{\gamma}^{p^{\prime}}\left(\mathbb{R}_{+}\right)$(resp. from $L_{\gamma}^{p}\left(\mathbb{R}_{+}\right)$onto $L_{A}^{p^{\prime}}\left(\mathbb{R}_{+}\right)$) and we have

$$
\|\mathscr{F} f\|_{L_{\gamma}^{p^{\prime}}\left(\mathbb{R}_{+}\right)} \leqslant\|f\|_{L_{A}^{p}\left(\mathbb{R}_{+}\right)} ; \quad\|\mathscr{g} g\|_{L_{A}^{p^{\prime}}\left(\mathbb{R}_{+}\right)} \leqslant\|g\|_{L_{\gamma}^{p}\left(\mathbb{R}_{+}\right)}
$$

with $\left(1 / p^{\prime}\right)+(1 / p)=1$.

\subsection{Generalized Convolution}

Definition 7 (see [10]). The translation operator associated with the operator $\Delta_{A}$ is defined on $L_{A}^{1}\left(\mathbb{R}_{+}\right)$, by

$$
\forall x, y \geqslant 0 ; \quad \tau_{x} f(y)=\int_{0}^{\infty} f(z) w(x, y, z) A(z) d z,
$$

where $w$ is the function defined in the relation (9).
Proposition 8 (see [10]). For a suitable function $f$ on $\mathbb{R}_{+}$, we have

$$
\begin{aligned}
& \text { (i) } \tau_{x} f(y)=\tau_{y} f(x), \\
& \text { (ii) } \tau_{0} f(y)=f(y), \\
& \text { (iii) } \tau_{x} \tau_{y}=\tau_{y} \tau_{x} \\
& \text { (iv) } \tau_{x} \varphi_{\lambda}(y)=\varphi_{\lambda}(x) \varphi_{\lambda}(y), \\
& \text { (v) } \mathscr{F}\left(\tau_{x} f\right)(\lambda)=\varphi_{\lambda}(x) \mathscr{F}(f)(\lambda), \\
& \text { (vi) } \Delta_{A}\left(\tau_{x}\right) f=\tau_{x}\left(\Delta_{A} f\right) .
\end{aligned}
$$

Definition 9 (see [10]). For suitable functions $f$ and $g$, we define the convolution product $f *_{A} g$ by

$$
f *_{A} g(x)=\int_{\mathbb{R}_{+}} \tau_{x} f(y) g(y) A(y) d y,
$$

Remark 10. It is clear that this convolution product is both commutative and associative:

(i) $f *_{A} g=g *_{A} f$.

(ii) $\left(f *_{A} g\right) *_{A} h=f *_{A}\left(g *_{A} h\right)$.

Proposition 11 (see [10]). (i) Assume that $1 \leq p, q, r \leq \infty$ satisfy $(1 / p)+(1 / q)-1=1 / r$. Then, for every $f \in L_{A}^{p}\left(\mathbb{R}_{+}\right)$ and $g \in L_{A}^{q}\left(\mathbb{R}_{+}\right)$, we have $f *_{A} g \in L_{A}^{r}\left(\mathbb{R}_{+}\right)$, and

$$
\left\|f *_{A} g\right\|_{L_{A}^{r}\left(\mathbb{R}_{+}\right)} \leq C\|f\|_{L_{A}^{p}\left(\mathbb{R}_{+}\right)}\|g\|_{L_{A}^{q}\left(\mathbb{R}_{+}\right)} .
$$

(ii) If $\rho>0$ and $1 \leq p<q \leq 2$, then

$$
L_{A}^{p}\left(\mathbb{R}_{+}\right) *{ }_{A} L_{A}^{q}\left(\mathbb{R}_{+}\right) \hookrightarrow L_{A}^{q}\left(\mathbb{R}_{+}\right)
$$

(iii) If $\rho>0$ and $2<p, q<\infty$ such that $q / 2 \leq p<q$, then

$$
L_{A}^{p}\left(\mathbb{R}_{+}\right) *_{A} L_{A}^{q^{\prime}}\left(\mathbb{R}_{+}\right) \hookrightarrow L_{A}^{q}\left(\mathbb{R}_{+}\right)
$$

where $q^{\prime}$ is the conjugate exponent of $q$. then

(iv) If $\rho>0$ and $1<p<2$ such that $p<q \leq p /(2-p)$,

$$
L_{A}^{p}\left(\mathbb{R}_{+}\right) *_{A} L_{A}^{p}\left(\mathbb{R}_{+}\right) \hookrightarrow L_{A}^{q}\left(\mathbb{R}_{+}\right)
$$

Proposition 12. For $f \in L_{A}^{2}\left(\mathbb{R}_{+}\right)$and $g \in L_{A}^{p}\left(\mathbb{R}_{+}\right)$, with $1 \leq$ $p<2$ we have

$$
\mathscr{F}\left(f *_{A} g\right)=\mathscr{F}(f)(\lambda) \mathscr{F}(g)(\lambda) .
$$

Proposition 13. Let $f, g \in L_{A}^{2}\left(\mathbb{R}_{+}\right)$. Then $f *_{A} g \in L_{A}^{2}\left(\mathbb{R}_{+}\right)$if and only if $\mathscr{F}(f) \mathscr{F}(g)$ belongs to $L_{A}^{2}\left(\mathbb{R}_{+}\right)$, and in this case we have

$$
\mathscr{F}\left(f *_{A} g\right)=\mathscr{F}(f) \mathscr{F}(g) .
$$

Definition 14. The generalized Fourier transform of a distribution $\tau$ in $\left(\mathcal{S}_{*}^{2}\right)^{\prime}(\mathbb{R})$ is defined by

$$
\langle\mathscr{F}(\tau), \phi\rangle=\left\langle\tau, \mathscr{F}^{-1}(\phi)\right\rangle, \quad \forall \phi \in \mathcal{S}_{*}(\mathbb{R})
$$


Proposition 15. The generalized Fourier transform $\mathscr{F}$ is a topological isomorphism from $\left(\mathcal{S}_{*}^{2}\right)^{\prime}(\mathbb{R})$ onto $\mathcal{S}_{*}^{\prime}(\mathbb{R})$.

Let $\tau$ be in $\left(\mathcal{S}_{*}^{2}\right)^{\prime}\left(\mathbb{R}_{+}\right)$. We define the distribution $\Delta_{A} \tau$, by

$$
\left\langle\Delta_{A} \tau, \psi\right\rangle=\left\langle\tau, \Delta_{A} \psi\right\rangle, \quad \forall \psi \in \mathcal{S}_{*}^{2}\left(\mathbb{R}_{+}\right) .
$$

This distribution satisfy the following property:

$$
\mathscr{F}\left(\Delta_{A} \tau\right)=-y^{2} \mathscr{F}(\tau) .
$$

\section{Sobolev-Type Spaces on the Dual of the Chébli-Trimèche Hypergroup}

Definition 16. Let $s \in \mathbb{R}$ and $p \in[1, \infty]$. We define the Sobolev-type spaces $W_{A}^{s, p}\left(\mathbb{R}_{+}\right)$as the set of tempered distributions $u \in S_{*}^{\prime}(\mathbb{R})$ such that

$$
\left(1+x^{2}\right)^{s} \mathscr{J}(u) \in L_{A}^{p}\left(\mathbb{R}_{+}\right) .
$$

We provide the space $W_{A}^{s, p}\left(\mathbb{R}_{+}\right)$with the norm:

$$
\|u\|_{W_{A}^{s, p}\left(\mathbb{R}_{+}\right)}=\left\|\left(1+x^{2}\right)^{s} \mathscr{J}(u)\right\|_{L_{A}^{p}\left(\mathbb{R}_{+}\right)} .
$$

In the sequel, we will give some properties of the space $W_{A}^{s, p}\left(\mathbb{R}_{+}\right)$.

Proposition 17. Let $s \in \mathbb{R}$. The space $\mathcal{S}_{*}(\mathbb{R})$ is dense in $W_{A}^{s, p}\left(\mathbb{R}_{+}\right)$, for

$$
p \in \begin{cases}{[1, \infty),} & \text { if } \rho=0 \\ {[2, \infty),} & \text { if } \rho>0 .\end{cases}
$$

Proof. Firstly, we want to prove that the space $\mathcal{S}_{*}(\mathbb{R})$ is a subset of $W_{A}^{s, p}\left(\mathbb{R}_{+}\right)$. Indeed, let $f \in \mathcal{S}_{*}(\mathbb{R})$. By Proposition 3 (i) the function $\left(1+x^{2}\right)^{s} \mathscr{J}(f) \in \mathcal{S}_{*}^{2}(\mathbb{R})$. Thus, using Remark 5 , we deduce the claim. Now, we prove the density. Let $f \in$ $W_{A}^{s, p}\left(\mathbb{R}_{+}\right)$. Then, from the density of $D_{*}\left(\mathbb{R}_{+}\right)$in $L_{A}^{p}\left(\mathbb{R}_{+}\right)$, we deduce the existence of a sequence $\left(g_{n}\right)_{n}$ in $D_{*}\left(\mathbb{R}_{+}\right)$such that

$$
\lim _{n \rightarrow \infty}\left\|\left(1+x^{2}\right)^{s} \mathscr{J}(f)-g_{n}\right\|_{L_{A}^{p}\left(\mathbb{R}_{+}\right)}=0 .
$$

On the other hand, according to Proposition 3(i), for all $n \in$ $\mathbb{N}$, the function $f_{n}=\mathscr{F}\left(\left(1+x^{2}\right)^{-s} g_{n}\right)$ is in $\mathcal{S}_{*}(\mathbb{R})$ and we have

$$
\left\|f-f_{n}\right\|_{W_{A}^{s, p}\left(\mathbb{R}_{+}\right)}=\left\|\left(1+x^{2}\right)^{s} \mathscr{J}(f)-g_{n}\right\|_{L_{A}^{p}\left(\mathbb{R}_{+}\right)} .
$$

Therefore, the result follows by combining (38) and (39).

Proposition 18. (i) Let $1 \leq p<\infty$ and let $s_{1}$ and $s_{2}$ in $\mathbb{R}$ such that $s_{2} \geq s_{1}$ then

$$
W_{A}^{s_{2}, p}\left(\mathbb{R}_{+}\right) \hookrightarrow W_{A}^{s_{1}, p}\left(\mathbb{R}_{+}\right) .
$$

(ii) Let $1 \leq p<\infty$, and let $s_{1}$, s and $s_{2}$ be three real numbers: $s_{1}<s<s_{2}$. Then, for all $\varepsilon>0$, there exists a nonnegative constant $C_{\varepsilon}$ such that for all $u$ in $W_{A}^{s, p}\left(\mathbb{R}_{+}\right)$

$$
\|u\|_{W_{A}^{s, p}\left(\mathbb{R}_{+}\right)} \leq C_{\varepsilon}\|u\|_{W_{A}^{s_{1}, p}\left(\mathbb{R}_{+}\right)}+\varepsilon\|u\|_{W_{A}^{s_{2}, p}\left(\mathbb{R}_{+}\right)} .
$$

Proof. (i) is clear.

(ii) We consider $s=(1-t) s_{1}+t s_{2}$, with $\left.t \in\right] 0,1[$. Moreover it is easy to see

$$
\|u\|_{W_{A}^{s, p}\left(\mathbb{R}_{+}\right)} \leq\|u\|_{W_{A}^{s_{1}, p}\left(\mathbb{R}_{+}\right)}^{1-t}\|u\|_{W_{A}^{s_{2}, p}\left(\mathbb{R}_{+}\right)}^{t} .
$$

Thus,

$$
\begin{aligned}
\|u\|_{W_{A}^{s, p}\left(\mathbb{R}_{+}\right)} & \leq\left(\varepsilon^{-t /(1-t)}\|u\|_{W_{A}^{s_{1}, p}\left(\mathbb{R}_{+}\right)}\right)^{1-t}\left(\varepsilon\|u\|_{W_{A}^{s_{2}, p}\left(\mathbb{R}_{+}\right)}\right)^{t} \\
& \leq \varepsilon^{-t /(1-t)}\|u\|_{W_{A}^{s_{1}, p}\left(\mathbb{R}_{+}\right)}+\varepsilon\|u\|_{W_{A}^{s_{2}, p}\left(\mathbb{R}_{+}\right)} .
\end{aligned}
$$

Hence, the proof is completed for $C_{\varepsilon}=\varepsilon^{-t /(1-t)}$.

Proposition 19. (i) Let $p \in[1, \infty]$ if $\rho=0$ and $p$ in $[1,2]$, if $\rho>0$. The space $W_{A}^{s, p}\left(\mathbb{R}_{+}\right)$provided with the norm $\|\cdot\|_{W_{A}^{s, p}\left(\mathbb{R}_{+}\right)}$ is a Banach space.

(ii) Let $s, t \in \mathbb{R}$ and $p \in[1, \infty]$. Then, the operator $(I+$ $\left.\mathscr{D}_{A}\right)^{t}$ defined, on $\mathcal{S}_{*}^{\prime}(\mathbb{R})$, by

$$
\left(I+\mathscr{D}_{A}\right)^{t} u=\mathscr{F}\left(\left(1+x^{2}\right)^{t} \mathscr{J}(u)\right)
$$

is an isometric isomorphism from $W_{A}^{s, p}\left(\mathbb{R}_{+}\right)$onto $W_{A}^{s-t, p}\left(\mathbb{R}_{+}\right)$. Moreover, for all $u \in W_{A}^{s, p}\left(\mathbb{R}_{+}\right), p \in[1,2]$, and for all $t \leq s$, the function

$$
x \longrightarrow\left(I+\mathscr{D}_{A}\right)^{t} u(x)
$$

belongs to the space $L_{\gamma}^{p^{\prime}}\left(\mathbb{R}_{+}\right)$, with $(1 / p)+\left(1 / p^{\prime}\right)=1$.

Proof. (i) Let $\left(f_{m}\right)_{m \in \mathbb{N}}$ be a Cauchy sequence in $W_{A}^{s, p}\left(\mathbb{R}_{+}\right)$. Then $\left(\left(1+x^{2}\right)^{s} \mathscr{J}\left(f_{m}\right)\right)_{m \in \mathbb{N}}$ is a Cauchy sequence in $L_{A}^{p}\left(\mathbb{R}_{+}\right)$. But $L_{A}^{p}\left(\mathbb{R}_{+}\right)$is complete, so, there exists a function $g$ such that $\left(1+x^{2}\right)^{s} g \in L_{A}^{p}\left(\mathbb{R}_{+}\right)$and

$$
\lim _{m \rightarrow \infty}\left\|\left(1+x^{2}\right)^{s} \mathscr{J}\left(f_{m}\right)-\left(1+x^{2}\right)^{s} g\right\|_{L_{A}^{p}\left(\mathbb{R}_{+}\right)}=0 .
$$

But since $p \in[1, \infty]$ when $\rho=0$ and $p \in[1,2]$ when $\rho>0$, then $g \in\left(S_{*}^{2}\right)^{\prime}(\mathbb{R})$ and consequently $f=\mathscr{F}(g) \in S_{*}^{\prime}(\mathbb{R})$. This implies that $f \in W_{A}^{s, p}\left(\mathbb{R}_{+}\right)$and from relation (46), we get

$$
\lim _{m \rightarrow \infty}\left\|f_{m}-f\right\|_{W_{A}^{s, p}\left(\mathbb{R}_{+}\right)}=0 .
$$

This achieves the proof of (i).

(ii) Let $u \in W_{A}^{s, p}\left(\mathbb{R}_{+}\right)$. By remarking that $\mathscr{J}$ is an isomorphism from $S_{*}^{\prime}(\mathbb{R})$ onto $\left(S_{*}^{2}(\mathbb{R})\right)^{\prime}$ and using the fact that

$$
\begin{gathered}
\left(1+x^{2}\right)^{s-t} \mathscr{J}\left(\left(I+\mathscr{D}_{A}\right)^{t} u\right)=\left(1+x^{2}\right)^{s} \mathscr{J}(u), \\
\left(I+\mathscr{D}_{A}\right)^{t} \circ\left(I+\mathscr{D}_{A}\right)^{-t}(u)=\left(I+\mathscr{D}_{A}\right)^{-t} \circ\left(I+\mathscr{D}_{A}\right)^{t}(u)=u,
\end{gathered}
$$

we deduce the first part of (ii). Now, let $u \in W_{A}^{s, p}\left(\mathbb{R}_{+}\right), p \in$ $[1,2]$. Then, for all $t \leq s$, the function

$$
x \longrightarrow\left(1+x^{2}\right)^{t} \mathscr{J}(u)
$$

belongs to the space $L_{A}^{p}\left(\mathbb{R}_{+}\right)$. Therefore, we obtain the second part of (ii) by using inequality (23). 
In the following, we prove a Hardy-Littlewood-Paley type inequality for the transform $\mathscr{J}$.

Proposition 20. (1) Let $p \in[1,2]$. Then, for $\rho=0$ and $\alpha>0$, there exists a positive constant $C_{1}$ such that for all $f \in L_{\gamma}^{p}\left(\mathbb{R}_{+}\right)$

$$
\int_{0}^{\infty} x^{2(\alpha+1)(p-2)}|\mathscr{J}(f)(x)|^{p} A(x) d x \leq C\|f\|_{L_{\gamma}^{p}\left(\mathbb{R}_{+}\right)}^{p} .
$$

(2) (i) For $\rho>0,1 \leq p<2, \alpha>-1 / 2$, and $\delta>0$, we have for all $s>2(\alpha+1)(1-(2 / p))$

$$
\begin{gathered}
\int_{0}^{\infty} x^{s p}|\mathscr{J}(f)(x)|^{p} A(x) d x \leq C(\delta, s, p)\|f\|_{L_{\gamma}^{p}\left(\mathbb{R}_{+}\right)}^{p}, \\
f \in L_{\gamma}^{p}\left(\mathbb{R}_{+}\right) \cap \mathscr{H}_{*, \delta}(\mathbb{C}) .
\end{gathered}
$$

(ii) For $\rho>0, p=2, \alpha>-1 / 2$, and $\delta>0$, we have (51) for all $s \geq 0$.

We start with the following lemma deduced from the hypothesis of the function $A$.

Lemma 21. (i) For any real $a>0$, there exist positive constants $C_{1}(a)$ and $C_{2}(a)$ such that for all $x \in[0, a]$,

$$
C_{1}(a) x^{2 \alpha+1} \leq A(x) \leq C_{2}(a) x^{2 \alpha+1} .
$$

(ii) For $\rho>0$,

$$
A(x) \sim e^{2 \rho x}, \quad(x \longrightarrow \infty) .
$$

(iii) For $\rho=0$,

$$
A(x) \sim x^{2 \alpha+1}, \quad(x \longrightarrow \infty) .
$$

Proof of Proposition 20. (1) Let $\rho=0$ and $\alpha>0$. Clearly, the operator $K$ defined on $L_{\gamma}^{p}\left(\mathbb{R}_{+}\right), 1 \leq p \leq 2$, by

$$
K(f)(x)=x^{2(\alpha+1)} \mathscr{J}(f)(x)
$$

is of strong type $(2,2)$ between the spaces $\left(\mathbb{R}_{+}, d \gamma(\lambda)\right)$ and $\left(\mathbb{R}_{+}, A(x) d x / x^{4(\alpha+1)}\right)$. Therefore, according to the Marcinkiewicz theorem (cf. [11]), to obtain the result, it suffices to show that $K$ is of weak type $(1,1)$ between the spaces under consideration. Indeed, using assertions (i) and (iii) of Lemma 21 and inequality (23), we obtain for all $\lambda>0$ and $f \in L_{\gamma}^{1}\left(\mathbb{R}_{+}\right)$

$$
\begin{aligned}
& \int_{\left\{x \in \mathbb{R}_{+}, K(f)(x)>\lambda\right\}} \frac{A(x) d x}{x^{4(\alpha+1)}} \\
& \leq C \int_{\left\{x \in \mathbb{R}_{+}, x^{2(\alpha+1)}\|\mathcal{F}(f)\|_{L_{A}^{\infty}\left(\mathbb{R}_{+}\right)}>\lambda\right\}} \frac{x^{2 \alpha+1} d x}{x^{4(\alpha+1)}} \\
& \leq C \int_{\left\{x \in \mathbb{R}_{+}, x^{2(\alpha+1)}\|f\|_{L_{\gamma}^{1}\left(\mathbb{R}_{+}\right)}>\lambda\right\}} \frac{d x}{x^{2 \alpha+3}} \\
& \quad=C \int_{\left(\lambda /\|f\|_{L_{\gamma}^{1}\left(\mathbb{R}_{+}\right)}\right)^{1 / 2(\alpha+1)}} \frac{d x}{x^{2 \alpha+3}} \\
& \leq C \frac{\|f\|_{L_{\gamma}^{1}\left(\mathbb{R}_{+}\right)}}{\lambda},
\end{aligned}
$$

and the desired result follows.
(2) Let $\rho>0, \alpha>-1 / 2$, and $\delta>0$. According to Proposition 3(ii), for all $f \in L_{\gamma}^{p}\left(\mathbb{R}_{+}\right) \cap \mathscr{H}_{*, \delta}(\mathbb{C})$, it follows that $\operatorname{supp}(\mathscr{J}(f)) \subseteq[-\delta, \delta]$.

(i) If $p \in[1,2)$ and $s>2(\alpha+1)(1-(2 / p))$, one can easily see by using Holder inequality, Lemma 21(i), and inequality (23) that for $f \in L_{\gamma}^{p}\left(\mathbb{R}_{+}\right) \cap \mathscr{H}_{*, \delta}(\mathbb{C})$

$$
\begin{gathered}
\left(\int_{0}^{\infty} x^{s p}|\mathcal{J}(f)(x)|^{p} A(x) d x\right)^{1 / p} \\
\leq C(\delta, s, p)\left\|1_{[0, \delta]} \mathscr{J}(f)\right\|_{L_{A}^{p^{\prime}}\left(\mathbb{R}_{+}\right)} \\
\leq C(\delta, s, p)\|f\|_{L_{\gamma}^{p}\left(\mathbb{R}_{+}\right)} .
\end{gathered}
$$

(ii) If $p=2$, then by virtue of Plancherel Theorem for the transform $\mathscr{J}$, we deduce that for all $s \geq 0$,

$$
\begin{gathered}
\int_{0}^{\infty} x^{2 s}|\mathscr{J}(f)(x)|^{2} A(x) d x \leq \delta^{2 s}\|f\|_{2, \gamma}^{2}, \\
f \in L_{\gamma}^{2}\left(\mathbb{R}_{+}\right) \cap \mathscr{H}_{*, \delta}(\mathbb{C}) .
\end{gathered}
$$

This completes the proof of the proposition.

Proposition 22. (1) Let $p \in(1,2]$. Then for $\rho=0, \alpha>0$, and $s \leq(\alpha+1)(1-(2 / p))$

$$
L_{\gamma}^{p}\left(\mathbb{R}_{+}\right) \hookrightarrow W_{A}^{s, p}\left(\mathbb{R}_{+}\right) .
$$

and we have

$$
\|f\|_{W_{A}^{s, p}\left(\mathbb{R}_{+}\right)} \leq C\|f\|_{L_{\gamma}^{p}\left(\mathbb{R}_{+}\right)}, \quad f \in L_{\gamma}^{p}\left(\mathbb{R}_{+}\right)
$$

(2) (i) Let $p \in[1,2)$. Then for $\rho>0, \alpha>-1 / 2$, and $\delta>0$, we have for all $s>(\alpha+1)(1-(2 / p))$

$$
\begin{gathered}
L_{\gamma}^{p}\left(\mathbb{R}_{+}\right) \cap \mathscr{H}_{*, \delta}(\mathbb{C}) \hookrightarrow W_{A}^{s, p}\left(\mathbb{R}_{+}\right), \\
\|f\|_{W_{A}^{s, p}\left(\mathbb{R}_{+}\right)} \leq C\|f\|_{L_{\gamma}^{p}\left(\mathbb{R}_{+}\right)}, \quad f \in L_{\gamma}^{p}\left(\mathbb{R}_{+}\right) \cap \mathscr{H}_{*, \delta}(\mathbb{C}) .
\end{gathered}
$$

(ii) For $p=2$. Then for $\rho>0, \alpha>-1 / 2$, and $\delta>0$, we have for all $s \geq 0$,

$$
\begin{gathered}
W_{A}^{s, 2}\left(\mathbb{R}_{+}\right) \subset L_{\gamma}^{2}\left(\mathbb{R}_{+}\right) \cap \mathscr{H}_{*, \delta}(\mathbb{C}) \\
\|f\|_{W_{A}^{s, 2}\left(\mathbb{R}_{+}\right)} \leq C\|f\|_{2, \gamma}, \quad f \in L_{\gamma}^{2}\left(\mathbb{R}_{+}\right) \cap \mathscr{H}_{*, \delta}(\mathbb{C}) .
\end{gathered}
$$

Proof. (1) The result follows from Proposition 20(1) and the fact that, for all $s \leq(\alpha+1)(1-(2 / p))$,

$$
\left(1+x^{2}\right)^{s} \leq x^{2(\alpha+1)(1-(2 / p))}, \quad x \in(0, \infty)
$$

(2) (i) If $p \in[1,2)$ and using the fact that, for all $s \in \mathbb{R}$,

$$
\left(1+x^{2}\right)^{s} \leq C\left(x^{2 s}+1\right), \quad x \in(0, \infty),
$$


it follows, from Holder inequality, that for all $\delta>0$ and $s>$ $(\alpha+1)(1-(2 / p))$

$$
\begin{aligned}
\|f\|_{W_{A}^{s, p}\left(\mathbb{R}_{+}\right)} \leq & C(\delta, s, p) \\
\times & {\left[\left(\int_{0}^{\infty} x^{2 s p}|\mathscr{J}(f)(x)|^{p} A(x) d x\right)^{1 / p}\right.} \\
& \left.+\left\|1_{[0, \delta]} \mathscr{J}(f)\right\|_{L_{A}^{p^{\prime}}\left(\mathbb{R}_{+}\right)}\right] .
\end{aligned}
$$

Thus, we deduce the result using Proposition 20(2) and inequality (23).

(ii) By virtue of (64), we obtain the result, for $p=2$, from Plancherel Theorem and Proposition 20(2).

Proposition 23. Let s be a non negative real number. Then, we have

(i) For $\rho \geq 0, W_{A}^{s, 1}\left(\mathbb{R}_{+}\right) \cap L_{A}^{2}\left(\mathbb{R}_{+}\right) \subset C_{*}^{2 m}\left(\mathbb{R}_{+}\right), m \in \mathbb{N}$; $m \leq s$.

(ii) If $\rho=0, W_{A}^{s, 2}\left(\mathbb{R}_{+}\right) \subset C_{*}^{2 m}\left(\mathbb{R}_{+}\right), m \in \mathbb{N}$; $m+(1 / 2)(\alpha+$ 1) $<s$,

where $C_{*}^{k}\left(\mathbb{R}_{+}\right)$is the space of even functions with class $C^{k}$ on $\mathbb{R}$.

Proof. (i) Let $u$ be in $W_{A}^{s, 1}\left(\mathbb{R}_{+}\right) \cap L_{A}^{2}\left(\mathbb{R}_{+}\right)$with $s \in \mathbb{R}_{+}$. It is clear that $\mathscr{J}(u)$ belongs to $L_{A}^{1}\left(\mathbb{R}_{+}\right) \cap L_{A}^{2}\left(\mathbb{R}_{+}\right)$.

Thus, from (14) and Proposition 4(ii), we have

$$
u(\lambda)=\int_{\mathbb{R}_{+}} \mathscr{J}(u)(x) \varphi_{\lambda}(x) A(x) d x, \quad \text { a.e. } \lambda \in \mathbb{R}_{+}
$$

We identify $u$ with the second member, then we deduce that $u$ belongs to $C_{*}(\mathbb{R})$ and the injection of $W_{A}^{s, 1}\left(\mathbb{R}_{+}\right)$into $C_{*}(\mathbb{R})$ is continuous.

Now, let $u$ be in $W_{A}^{s, 1}\left(\mathbb{R}_{+}\right)$with $s \in \mathbb{R}_{+}$such that $s>m$ with $m \in \mathbb{N} \backslash\{0\}$. From (12), for all $x, \lambda \in \mathbb{R}_{+}$, and $n \in \mathbb{N}$ such that $n \leq p$, we have

$$
\left|D_{\lambda}^{n} \varphi_{\lambda}(x)\right| \leq x^{n}
$$

Using the same method as for $m=0$ and the derivation theorem under the integral sign, we deduce that

$$
\begin{gathered}
\forall x \in \mathbb{R}_{+}, \\
D_{\lambda}^{n} u(\lambda)=\int_{\mathbb{R}_{+}} \mathscr{J}(u)(x) D_{\lambda}^{n} \varphi_{\lambda}(x) A(x) d x .
\end{gathered}
$$

Then, for all $n \in \mathbb{N}$ such that $n \leq 2 m, D_{\lambda}^{n} u$ belongs to $C_{*}(\mathbb{R})$. Thus, $u$ is in $C_{*}^{2 m}(\mathbb{R})$ and the injection of $W_{A}^{s, 1}\left(\mathbb{R}_{+}\right)$ into $C_{*}^{2 m}(\mathbb{R})$ is continuous.

(ii) If $\rho=0$ and $u$ in $W_{A}^{s, 2}\left(\mathbb{R}_{+}\right)$; then using assertions (i) and (iii) of Lemma 21 and Holder inequality, we deduce that for all $m \in \mathbb{N} ; m+(1 / 2)(\alpha+1)<s$, the function $u$ belongs to $W_{A}^{m, 1}\left(\mathbb{R}_{+}\right) \cap L_{A}^{2}\left(\mathbb{R}_{+}\right)$.

Therefore, (ii) follows from (i).
Proposition 24. For $1 \leq p<\infty$ and $s \in \mathbb{R}$, the space $W_{A}^{s, p}\left(\mathbb{R}_{+}\right)$is separable.

Proof. Let $p \in[1, \infty)$. It is well known that $L_{A}^{p}\left(\mathbb{R}_{+}\right)$is separable. More precisely, the set

$$
E=\left\{A^{-1 / p}(x) \sum_{\text {finite }} \alpha_{i} 1_{] a_{i}, b_{i}[}(x) ; \alpha_{i}, a_{i}, b_{i} \in \mathbb{Q}\right\}
$$

is countable and dense in $L_{A}^{p}\left(\mathbb{R}_{+}\right)$. Thus, for all $u \in W_{A}^{s, p}\left(\mathbb{R}_{+}\right)$, $s \in \mathbb{R}$, there exists a sequence $\left(u_{n}\right)_{n \in \mathbb{N}}$ in $E$ such that

$$
\lim _{n \rightarrow \infty}\left\|u_{n}-\left(1+x^{2}\right)^{s} \mathscr{J}(u)\right\|_{L_{A}^{p}\left(\mathbb{R}_{+}\right)}=0
$$

On the other hand, for all $n \in \mathbb{N}, u_{n} \in\left(L_{A}^{1} \cap L_{A}^{p}\right)\left(\mathbb{R}_{+}\right)$, and so $u_{n} \in\left(S_{*}^{2}\right)^{\prime}\left(\mathbb{R}_{+}\right)$. Therefore, for all $n \in \mathbb{N}$, there exists $v_{n} \in$ $W_{A}^{s, p}\left(\mathbb{R}_{+}\right)$such that $u_{n}=\left(1+x^{2}\right)^{s} \mathscr{J}\left(v_{n}\right)$. Hence, from $(70)$, we obtain

$$
\lim _{n \rightarrow \infty}\left\|v_{n}-u\right\|_{W_{A}^{s, p}\left(\mathbb{R}_{+}\right)}=0
$$

This implies that $F=\left\{\mathscr{F}\left(\left(1+x^{2}\right)^{-s} f\right): f \in E\right\}$ is countable and dense in $W_{A}^{s, p}\left(\mathbb{R}_{+}\right)$and the proposition is proved.

3.1. Reillich-Type Theorem. In this subsection, using Hahn Banach's and Riesz's theorems [12, 13], we describe the dual space $\left(W_{A}^{s, p}\left(\mathbb{R}_{+}\right)\right)^{*}$ of $W_{A}^{s, p}\left(\mathbb{R}_{+}\right)$. We prove also that a compact imbedding theorem and a Reillich-type theorem are established. We need firstly the following lemmas.

Lemma 25. Let $s \in \mathbb{R}$ and $x, t \geq 0$. For all positive continuous function $f$, we have

$$
\left(1+x^{2}\right)^{s}\left|\tau_{x} f(t)\right| \leq 2^{|s|}\left(1+t^{2}\right)^{s}\left|\tau_{x}\left[\left(1+y^{2}\right)^{|s|} f\right](t)\right| .
$$

Proof. The result follows by using the following classical Peetre's inequality:

$$
\left(1+x^{2}\right)^{s} \leq 2^{|s|}\left(1+t^{2}\right)^{s}\left(1+|x-t|^{2}\right)^{|s|},
$$

and the fact that the kernel $W(x, t, \cdot)$ is positive with support in $[|x-t|, x+t]$.

Lemma 26. For all $u \in \mathcal{S}_{*}^{\prime}(\mathbb{R})$ and $\phi \in \mathcal{S}_{*}(\mathbb{R})$, we have

$$
\mathscr{J}(\Phi u)=\mathscr{J}(u) *_{A} \mathscr{J}(\Phi),
$$

where, for $S$ in $\left(S_{*}^{2}\right)^{\prime}(\mathbb{R})$ and $\psi$ in $S_{*}^{2}(\mathbb{R})$, the function $S * \psi$ is the generalized convolution product of the distribution $S$ and the function $\psi$ defined by

$$
S *_{A} \psi(x)=\left\langle S, \tau_{x}(\psi)\right\rangle .
$$

Proof. For all $S \in\left(S_{*}^{2}\right)^{\prime}(\mathbb{R})$ and $\psi \in S_{*}^{2}(\mathbb{R})$, the function $S * \psi$ belongs to $\left(S_{*}^{2}\right)^{\prime}(\mathbb{R})(\mathrm{cf}$. [4]) and we have

$$
\begin{gathered}
\left\langle S *_{A} \psi, \varphi\right\rangle=\left\langle S, \psi *_{A} \phi\right\rangle, \quad \phi \in S_{*}^{2}(\mathbb{R}), \\
\mathscr{F}\left(S *_{A} \psi\right)=\mathscr{F}(S) \mathscr{F}(\psi) .
\end{gathered}
$$


Therefore, the result follows by using the fact that $\mathscr{F}$ is an isomorphism from $S_{*}^{2}(\mathbb{R})$ (resp. $\left(S_{*}^{2}\right)^{\prime}(\mathbb{R})$ ) onto $\mathcal{S}_{*}(\mathbb{R})$ (resp. $\left.\mathcal{S}_{*}^{\prime}(\mathbb{R})\right)$.

Theorem 27. Let $s \in \mathbb{R}, \Phi \in \mathcal{S}_{*}(\mathbb{R})$, and $1 \leq q \leq r \leq \infty$.

(i) If $\rho=0$, then the mapping

$$
\begin{aligned}
W_{A}^{s, q}\left(\mathbb{R}_{+}\right) & \longrightarrow W_{A}^{s, r}\left(\mathbb{R}_{+}\right) \\
u & \longmapsto \Phi u
\end{aligned}
$$

is continuous.

(ii) If $\rho>0$. We have the same result as in (i) if $1 / 2 \leq$ $(1 / q)-(1 / r) \leq 1$.

(iii) If $\rho>0$ and $2<q<\infty$, then the mapping

$$
\begin{aligned}
W_{A}^{s, q^{\prime}}\left(\mathbb{R}_{+}\right) & \longrightarrow W_{A}^{s, q}\left(\mathbb{R}_{+}\right) \\
u & \longmapsto \Phi u
\end{aligned}
$$

is continuous.

(iv) Let $\rho>0, s \geq 0$, and $q \leq 2 \leq r$. Then, the mapping

$$
\begin{aligned}
W_{A}^{s, q}\left(\mathbb{R}_{+}\right) & \longrightarrow W_{A}^{0, r}\left(\mathbb{R}_{+}\right) \\
u & \longmapsto \Phi u
\end{aligned}
$$

is continuous.

Proof. (1) (i) According to Lemmas 25 and 26, we have, for $\Phi \in \mathcal{S}_{*}(\mathbb{R})$ and $u \in W_{A}^{s, p}\left(\mathbb{R}_{+}\right)$

$$
\begin{aligned}
\left|\left(1+x^{2}\right)^{s} \mathscr{J}(\Phi u)(x)\right| & \mid\left\langle\left(1+t^{2}\right)^{s} \mathscr{J}(u),\right. \\
& \left.\left(1+t^{2}\right)^{-s}\left(1+x^{2}\right)^{s} \tau_{x}(\mathscr{J}(\Phi))\right\rangle_{L_{A}^{2}\left(\mathbb{R}_{+}\right) \mid} \mid \\
\leq & 2^{|s|}\left[\left(1+t^{2}\right)^{s}|\mathscr{J}(u)|\right] *\left[\left(1+t^{2}\right)^{|s|}|\mathscr{J}(\Phi)|\right](x) .
\end{aligned}
$$

On the other hand, from the hypothesis on $q$ and $r$, there exists

$$
p \in[1, \infty]: \frac{1}{p}+\frac{1}{q}-1=\frac{1}{r} .
$$

Thus, using Proposition 11(i), we obtain the result.

(ii) As $\Phi \in \mathcal{S}_{*}(\mathbb{R})$, it is easy to see that $\Phi \in W_{A}^{|s|, p}\left(\mathbb{R}_{+}\right)$ for $2 \leq p \leq \infty$. Moreover, we proceed as above and using Proposition 11(i), we obtain

$$
\|\Phi u\|_{W_{A}^{s, r}\left(\mathbb{R}_{+}\right)} \leq 2^{|s|}\|u\|_{W_{A}^{s, q}\left(\mathbb{R}_{+}\right)}\|\Phi\|_{W_{A}^{|s|, p}\left(\mathbb{R}_{+}\right)},
$$

for

$$
p \in[2, \infty]: \frac{1}{p}+\frac{1}{q}-1=\frac{1}{r} .
$$

Hence, using the fact that the imbedding $\delta_{*}(\mathbb{R}) \hookrightarrow$ $W_{A}^{|s|, p}\left(\mathbb{R}_{+}\right)$is continuous, the desired result follows. (iii) Let $p \in(\max (2, q / 2), q)$. Then $\Phi \in W_{A}^{|s|, p}\left(\mathbb{R}_{+}\right)$. Therefore, from (80) and using Proposition 11(iii), we deduce the result.

(iv) Using (23) and Holder's inequality, we obtain, for all $\Phi \in \mathcal{S}_{*}(\mathbb{R})$ and $u \in W_{A}^{s, q}\left(\mathbb{R}_{+}\right)$,

$$
\begin{aligned}
& \|\Phi u\|_{W_{A}^{0, r}\left(\mathbb{R}_{+}\right)} \\
& \leq\|\Phi u\|_{L_{\gamma}^{r^{\prime}\left(\mathbb{R}_{+}\right)}} \\
& \leq C \begin{cases}\|u\|_{L_{\gamma}^{q^{\prime}}\left(\mathbb{R}_{+}\right)}\|\Phi\|_{L_{\gamma}^{q r /(r-q)}\left(\mathbb{R}_{+}\right)}, & \text {for } r \neq q \\
\|u\|_{L_{\gamma}^{2}\left(\mathbb{R}_{+}\right)}\|\Phi\|_{L_{\gamma}^{\infty}\left(\mathbb{R}_{+}\right)}, & \text {for } r=q=2\end{cases} \\
& \leq C \begin{cases}\|u\|_{W_{A}^{s, q}\left(\mathbb{R}_{+}\right)}\|\Phi\|_{L_{\gamma}^{q r /(r-q)}\left(\mathbb{R}_{+}\right)}, & \text {for } r \neq q \\
\|u\|_{W_{A}^{s, 2}\left(\mathbb{R}_{+}\right)}\|\Phi\|_{L_{\gamma}^{\infty}\left(\mathbb{R}_{+}\right)}, & \text {for } r=q=2,\end{cases}
\end{aligned}
$$

where $q^{\prime}$ and $r^{\prime}$ are,respectively, the conjugates of $q$ and $r$. Therefore, we deduce the result by remarking that the embedding $\mathcal{S}_{*}(\mathbb{R}) \hookrightarrow L_{\gamma}^{p}\left(\mathbb{R}_{+}\right)$is continuous. This achieves the proof of theorem.

Now, we shall characterize the dual space $\left(W_{A}^{s, p}\left(\mathbb{R}_{+}\right)\right)^{*}$ of $W_{A}^{s, p}\left(\mathbb{R}_{+}\right)$.

Theorem 28. Let $p \in[1, \infty)$ when $\rho=0$ and $p \in[2, \infty)$ when $\rho>0$. The dual space $\left(W_{A}^{s, p}\left(\mathbb{R}_{+}\right)\right)^{*}$ of $W_{A}^{s, p}\left(\mathbb{R}_{+}\right)$can be identified with $W_{A}^{-s, p^{\prime}}\left(\mathbb{R}_{+}\right)$, where $q^{\prime}$ is the conjugate of $p$.

Proof. Let $p \in[1,+\infty)$ when $\rho=0$ and $p \in[2, \infty)$ when $\rho>0$. Then, for $u \in W_{A}^{-s, p^{\prime}}\left(\mathbb{R}_{+}\right)$, we have, for all $\varphi \in S_{*}(\mathbb{R})$,

$$
\begin{aligned}
|\langle u, \varphi\rangle| & =\left|\left\langle\left(1+x^{2}\right)^{-s} \mathscr{J}(u),\left(1+x^{2}\right)^{s} \mathscr{J}(\varphi)\right\rangle_{L_{A}^{2}\left(\mathbb{R}_{+}\right)}\right| \\
& \leq\|u\|_{W_{A}^{-s, p^{\prime}}{ }_{\left(\mathbb{R}_{+}\right)}\|\varphi\|_{W_{A}^{s, p}\left(\mathbb{R}_{+}\right)}}
\end{aligned}
$$

This proves, from the density of $S_{*}(\mathbb{R})$ in $W_{A}^{s, p}\left(\mathbb{R}_{+}\right)$, that $u$ admits a unique continuous extension to $W_{p}^{s}$.

Conversely, suppose that $u \in\left(W_{A}^{s, p}\left(\mathbb{R}_{+}\right)\right)^{*}$. Then the mapping

$$
\begin{gathered}
\mathscr{V}: L_{A}^{p}\left(\mathbb{R}_{+}\right) \longrightarrow \mathbb{C} \\
\psi \longmapsto\left\langle u, \mathcal{U}^{-1}(\psi)\right\rangle
\end{gathered}
$$

is continuous, where $\mathcal{U}$ is the isometric isomorphism from $\left(W_{A}^{s, p}\left(\mathbb{R}_{+}\right),\|\cdot\|_{W_{A}^{s, p}\left(\mathbb{R}_{+}\right)}\right)$into $\left(L_{A}^{p}\left(\mathbb{R}_{+}\right),\|\cdot\|_{L_{A}^{p}\left(\mathbb{R}_{+}\right)}\right)$, defined by

$$
\mathcal{U}(\varphi)=\left(1+x^{2}\right)^{s} \mathscr{J}(\varphi)
$$

with inverse

$$
\mathcal{U}^{-1}(\psi)=\mathscr{F}\left(\left(1+x^{2}\right)^{-s} \psi\right)
$$


Thus, and using the fact that $u \in S_{*}^{\prime}(\mathbb{R})$, there exists $C>0$ such that

$$
\begin{gathered}
\forall \psi \in D_{*}(\mathbb{R}), \\
|\mathscr{V}(\psi)|=\left|\left\langle\left(1+x^{2}\right)^{-s} \mathscr{J}(u), \psi\right\rangle_{L_{A}^{2}\left(\mathbb{R}_{+}\right)}\right| \leq C\|\psi\|_{p, v} .
\end{gathered}
$$

Hence, from the density of $D_{*}(\mathbb{R})$ in $L_{A}^{p}\left(\mathbb{R}_{+}\right)$and using Riez's theorem, we deduce that $u$ belongs to $W_{A}^{-s, p^{\prime}}\left(\mathbb{R}_{+}\right)$. This completes the proof of Theorem 28.

Proposition 29. Let $q \in(1, \infty)$ when $\rho=0$ and $q=2$ when $\rho>0$. Let $\Phi$ be in $\mathcal{S}_{*}(\mathbb{R})$ and $r \in[q, \infty)$. Then for all $s, t \in \mathbb{R}$ such that $t<s$ if $\rho=0$ and $t<0 \leq s$ if $\rho>0$, the mapping

$$
\begin{aligned}
W_{A}^{s, q}\left(\mathbb{R}_{+}\right) & \longrightarrow W_{A}^{t, r}\left(\mathbb{R}_{+}\right) \\
u & \mapsto \Phi u
\end{aligned}
$$

is compact.

Proof. Let $\left(u_{m}\right)_{m}$ be a sequence in $W_{A}^{s, q}\left(\mathbb{R}_{+}\right)$such that $\left\|u_{m}\right\|_{W_{A}^{s, q}\left(\mathbb{R}_{+}\right)} \leq 1$, for all $m \in \mathbb{N}$. Then, from Theorem 28, we deduce that $\left(u_{m}\right)_{m}$ can be regarded as a sequence in $\left(W_{A}^{-s, q^{\prime}}\left(\mathbb{R}_{+}\right)\right)^{*}$, with $(1 / q)+\left(1 / q^{\prime}\right)=1$, and using Holder inequality, we obtain for all $m \in \mathbb{N}$

$$
\begin{aligned}
& \left\|u_{m}\right\|_{\left(W_{A}^{-s, q^{\prime}}\left(\mathbb{R}_{+}\right)\right)^{*}}
\end{aligned}
$$

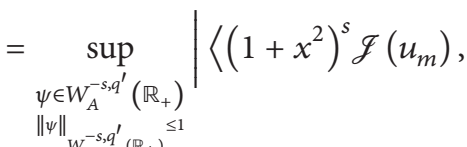

$$
\begin{aligned}
& \left.\left(1+x^{2}\right)^{-s} \mathscr{J}(\psi)\right\rangle_{L_{A}^{2}\left(\mathbb{R}_{+}\right)} \\
& \leq\left\|u_{m}\right\|_{W_{A}^{s, q}\left(\mathbb{R}_{+}\right)} \leq 1 .
\end{aligned}
$$

Therefore, by virtue of Propositions 24 and 19(i), $W_{A}^{-s, q^{\prime}}\left(\mathbb{R}_{+}\right)$ is a separable Banach space. Which implies, from Alaoglu theorem (cf. [14]), that there exists a subsequence $\left(u_{m_{k}}\right)_{k}$ weakly converging in $\left(W_{A}^{-s, q^{\prime}}\left(\mathbb{R}_{+}\right)\right)^{*}$. We denote by $u$ its weak limit. Using Lemma 26 , for all $k \in \mathbb{N}$, we have

$$
\begin{aligned}
\mathscr{J}\left[\Phi\left(u_{m_{k}}-u\right)\right](x) & =\left[\mathscr{J}\left(u_{m_{k}}-u\right) *_{A} \mathscr{J}(\Phi)\right](x) \\
& =\left\langle\mathscr{J}\left(u_{m_{k}}-u\right), \tau_{x}(\mathscr{J}(\Phi))\right\rangle \\
& =\left\langle u_{m_{k}}-u, \varphi_{(\cdot)}(x) \Phi\right\rangle .
\end{aligned}
$$

$\operatorname{But}\left(u_{m_{k}}-u\right)_{k}$ is weakly converging to zero in $\left(W_{A}^{-s, q^{\prime}}\left(\mathbb{R}_{+}\right)\right)^{*}$, then it follows that for all $x \in \mathbb{R} \lim _{k \rightarrow \infty} \mathscr{J}\left(\Phi \cdot\left(u_{m_{k}}-u\right)(x)=\right.$ 0 .
Now, according to Theorem 27 , for all $k \in \mathbb{N}, \Phi \cdot\left(u_{m_{k}}-u\right) \in$ $W_{A}^{s, \infty}\left(\mathbb{R}_{+}\right)$and using (82) we get

$\forall k \in \mathbb{N}, \quad \forall x \in \mathbb{R}$,

$$
\begin{aligned}
& \left|\left(1+x^{2}\right)^{s} \mathcal{J}\left(\Phi \cdot\left(u_{m_{k}}-u\right)\right)(x)\right| \\
& \quad \leq 2^{|s|}\left(1+\|u\|_{W_{A}^{s, q}\left(\mathbb{R}_{+}\right)}\right) \cdot\|\Phi\|_{W_{A}^{\mid s, q^{\prime}}\left(\mathbb{R}_{+}\right)} .
\end{aligned}
$$

Hence, by Lebesgue's theorem, we deduce that for all $R>0$,

$$
\begin{array}{r}
\lim _{k \rightarrow \infty} \int_{0}^{R}\left(1+x^{2}\right)^{r t}\left|\mathcal{F}\left(\Phi \cdot\left(u_{m_{k}}-u\right)\right)(x)\right|^{r} A(x) d x=0, \\
r \in[q, \infty) .
\end{array}
$$

On the other hand, for $\rho=0$ with $t<s, q \in(1, \infty)$, and $r \in[q, \infty)$, it follows from Theorem 27(i) that for $p \in[1, \infty]$ and satisfying $(1 / p)+(1 / q)-1=1 / r$, we have

$$
\begin{gathered}
\int_{R}^{\infty}\left(1+x^{2}\right)^{r t}\left|\mathcal{J}\left(\Phi \cdot\left(u_{m_{k}}-u\right)\right)(x)\right|^{r} A(x) d x \\
\leq \frac{\left\|\Phi \cdot\left(u_{m_{k}}-u\right)\right\|_{W_{A}^{s, r}\left(\mathbb{R}_{+}\right)}^{r}}{\left(1+R^{2}\right)^{r(s-t)}} \\
\leq C \frac{\left(1+\|u\|_{W_{A}^{s, q}\left(\mathbb{R}_{+}\right)}\right)^{r}\|\Phi\|_{W_{A}^{r s, p}\left(\mathbb{R}_{+}\right)}^{r} .}{\left(1+R^{2}\right)^{r(s-t)}} .
\end{gathered}
$$

And for $\rho>0$ with $t<0 \leq s, q=2$, and $r \in[2, \infty)$, we obtain from Theorem 27(iv)

$$
\begin{aligned}
& \int_{R}^{\infty}\left(1+x^{2}\right)^{r t}\left|\mathcal{g}\left(\Phi \cdot\left(u_{m_{k}}-u\right)\right)(x)\right|^{r} A(x) d x \\
& \leq \frac{\left\|\Phi \cdot\left(u_{m_{k}}-u\right)\right\|_{W_{A}^{0, r}\left(\mathbb{R}_{+}\right)}^{r}}{\left(1+R^{2}\right)^{-r t}} \\
& \quad \leq \begin{cases}C \frac{\left(1+\|u\|_{W_{A}^{s 2}\left(\mathbb{R}_{+}\right)}\right)^{r}\|\Phi\|_{L_{\gamma}^{r r /(r-2)}\left(\mathbb{R}_{+}\right)}^{r},}{\left(1+R^{2}\right)^{-r t}} & \text { for } r \neq 2 \\
C \frac{\left(1+\|u\|_{W_{A}^{s 2}\left(\mathbb{R}_{+}\right)}\right)^{2}\|\Phi\|_{L_{\gamma}^{\infty}\left(\mathbb{R}_{+}\right)}^{2}}{\left(1+R^{2}\right)^{-2 t}}, & \text { for } r=2 .\end{cases}
\end{aligned}
$$

Which implies that these last integrals, (95) and (96), tend to zero when $R$ tends to $\infty$ uniformly with respect to $k \in \mathbb{N}$ and so, by virtue of (94), we conclude that $\left(\Phi \cdot\left(u_{m_{k}}-u\right)\right)_{k}$ is strongly converging in $W_{A}^{t, r}\left(\mathbb{R}_{+}\right)$; that is, $\lim _{k \rightarrow \infty} \| \Phi .\left(u_{m_{k}}-\right.$ $u) \|_{W_{A}^{t, r}\left(\mathbb{R}_{+}\right)}=0$. This achieves the proof of Proposition 29.

Notation. Let $K$ be a compact contained in $\mathbb{R}$. We denote by $W_{A, K}^{s, q}\left(\mathbb{R}_{+}\right)$the subspace of $W_{A}^{s, q}\left(\mathbb{R}_{+}\right)$defined by

$$
W_{A, K}^{s, q}\left(\mathbb{R}_{+}\right)=\left\{u \in W_{A}^{s, q}\left(\mathbb{R}_{+}\right) ; \operatorname{supp} u \subset K\right\} .
$$


As a consequence of Proposition 29, with $\varphi \in \mathcal{S}_{*}(\mathbb{R})$ supported on a bounded set $V$ containing the compact $K$ and satisfying $\varphi=1$ on $K$, we deduce the main result of this section.

Theorem 30 (Reillich-type theorem). Let $q \in(1, \infty)$ when $\rho=0$ and $q=2$ when $\rho>0$. Let $K$ be a compact contained in $\mathbb{R}$ and let $r \in[q, \infty)$. Then for all $s, t \in \mathbb{R}$ such that $t<s$ if $\rho=0$ and $t<0 \leq s$ if $\rho>0$, the canonical imbedding $W_{A, K}^{s, q}\left(\mathbb{R}_{+}\right) \hookrightarrow W_{A, K}^{t, r}\left(\mathbb{R}_{+}\right)$is compact.

Using Reillich-type theorem, we prove the following inequalities.

Corollary 31. Let $K$ be a compact contained in $\mathbb{R}$ and $s \geq 0$. Then, for $q \in(1, \infty)$ when $\rho=0$ and $q=2$ when $\rho>0$, there exists $C>0$ such that, for all $u \in W_{A, K}^{s, q}\left(\mathbb{R}_{+}\right)$, we have

$$
\frac{1}{C}\|u\|_{W_{A}^{s, q}\left(\mathbb{R}_{+}\right)} \leq \dot{J}_{s}(u) \leq C\|u\|_{W_{A}^{s, q}\left(\mathbb{R}_{+}\right)},
$$

where $\dot{J}_{s}(u)=\left(\int_{0}^{\infty} x^{q s}|\mathscr{J}(u)(x)|^{q} A(x) d x\right)^{1 / q}$.

Proof. It is clear that, for all $u \in W_{A, K}^{s, q}\left(\mathbb{R}_{+}\right), \mathscr{J}(u) \in L_{A}^{q}\left(\mathbb{R}_{+}\right)$ and we have

$$
\dot{J}_{s}(u) \leq\|u\|_{W_{A}^{s, q}\left(\mathbb{R}_{+}\right)} .
$$

Now, let us prove the left hand side inequality. Suppose that for all positive integer $k$, there exists $u_{k} \in W_{A, K}^{s, q}\left(\mathbb{R}_{+}\right)$such that

$$
\frac{1}{k}\left\|u_{k}\right\|_{W_{A}^{s, q}\left(\mathbb{R}_{+}\right)}>\dot{J}_{s}\left(u_{k}\right) .
$$

Without loss of generality, one can suppose that $\left\|u_{k}\right\|_{W_{A}^{s, q}\left(\mathbb{R}_{+}\right)}=$ 1. Then we have

$$
\lim _{k \rightarrow \infty} \dot{J}_{s}\left(u_{k}\right)=0
$$

and, by using Reillich-type theorem, we deduce that there exists a subsequence $\left(u_{k_{n}}\right)_{n}$ of $\left(u_{k}\right)_{k}$ strongly converging in $W_{A}^{t, q}\left(\mathbb{R}_{+}\right), t<0$. Let $u$ be its strong limit. Therefore, by (101) and the fact that for $R>0$

$$
\begin{aligned}
& \left(\int_{0}^{R} x^{2 q s}|\mathcal{J}(u)(x)|^{q} A(x) d x\right)^{1 / q} \\
& \quad \leq\left(1+R^{2}\right)^{s-t}\left\|u-u_{k_{n}}\right\|_{W_{A}^{t, q}\left(\mathbb{R}_{+}\right)}+\dot{J}_{s}\left(u_{k_{n}}\right),
\end{aligned}
$$

it follows that $\dot{J}_{s}(u)=0$. This implies that $\mathscr{J}(u)=0$ and so $u=0$. On the other hand, there exists a positive constant $C$ such that

$$
\begin{aligned}
1= & \left\|u_{k_{n}}\right\|_{W_{A, K}^{s}\left(\mathbb{R}_{+}\right)} \\
\leq & C\left\{\left(\int_{0}^{1}\left|\left(1+x^{2}\right)^{t} \mathscr{J}\left(u_{k_{n}}\right)(x)\right|^{q} A(x) d x\right)^{1 / q}\right. \\
& \left.+\left(\int_{1}^{\infty}\left|x^{2 s} \mathscr{J}\left(u_{k_{n}}\right)(x)\right|^{q} A(x) d x\right)^{1 / q}\right\} . \\
\leq C & \left\{\left\|u_{k_{n}}\right\|_{W_{A}^{t, q}\left(\mathbb{R}_{+}\right)}+\dot{J}_{s}\left(u_{k_{n}}\right)\right\} .
\end{aligned}
$$

Hence, by tending $n$ to infinity and from (101), it follows that $1 \leq 0$ which is impossible. Thus, the required inequality is satisfied. Combining the left hand side inequality of (98) and (99), we obtain

$$
\frac{1}{C}\|u\|_{W_{A}^{s, q}\left(\mathbb{R}_{+}\right)} \leq \dot{J}_{s}(u) \leq C\|u\|_{W_{A}^{s, q}\left(\mathbb{R}_{+}\right)} .
$$

This completes the proof.

\section{Applications}

4.1. Weierstrass Transform on the Dual of the Chébli-Trimèche Hypergroup. This subsection is devoted to define and establish some properties for the Weierstrass transform $\mathscr{T}_{A}^{s}$, on the dual of the Chébli-Trimèche hypergroup, which we need later.

Definition 32. Let $s \geq 0$. We define the generalized Weierstrass transform of order $s$ on $S_{*}^{\prime}(\mathbb{R})$ as follows:

$$
\mathscr{T}_{A}^{s}(u)=\mathscr{F}\left(e^{-s\left(\lambda^{2}+\rho^{2}\right)} \mathscr{F}^{-1}(u)\right) .
$$

For $1 \leq p<\infty$, we denote by

$$
\mathscr{W}_{A}^{\mathcal{s}, p}\left(\mathbb{R}_{+}\right):=\left\{\phi \in \mathcal{S}_{*}^{\prime}(\mathbb{R}): \mathscr{T}_{A}^{s}(\phi) \in L_{\gamma}^{p}\left(\mathbb{R}_{+}\right)\right\}
$$

The norm in $\mathscr{W}_{A}^{s, p}\left(\mathbb{R}_{+}\right)$is given by

$$
\|\phi\|_{\mathscr{W}_{A}^{s, p}\left(\mathbb{R}_{+}\right)}=\left\|\mathscr{T}_{A}^{s}(\phi)\right\|_{L_{\gamma}^{p}\left(\mathbb{R}_{+}\right)} .
$$

Remark 33. Let $s>0$. For all $\lambda \in \mathbb{R}$, we have

$$
e^{-s\left(\lambda^{2}+\rho^{2}\right)}=\frac{1}{\left(\lambda^{2}+\rho^{2}\right)^{n}} \mathscr{F}\left(w_{n}(s, \cdot)\right)(\lambda),
$$

where $w_{n}(s, \cdot), n \in \mathbb{N}$, are the heat functions on the ChébliTrimèche hypergroup $\left(\mathbb{R}_{+}, *_{A}\right)$. In particular, $w_{n}(s, \cdot)=E_{s}$ is the Gaussian kernel on $\left(\mathbb{R}_{+}, *_{A}\right)$, see [15].

In the case of the Bessel-Kingman hypergroup (when the function $A$ is of the form $A(x)=x^{2 \alpha+1}$ and $\rho=0$ ), the Weierstrass transform associated with the Hankel transform is studied in [16]. For the classical Weierstrass transform, one can see [17-19].

In the following, we show some properties for $\mathscr{T}_{A}^{s}$ and $\mathscr{W}_{A}^{s, p}\left(\mathbb{R}_{+}\right)$.

Proposition 34. (i) Let $s, t \geq 0$. For all $f \in \mathcal{S}_{*}^{\prime}(\mathbb{R})$,

$$
\mathscr{T}_{A}^{s}\left(\mathscr{T}_{A}^{t}(f)\right)=\mathscr{T}_{A}^{s+t}(f), \quad \mathscr{T}_{A}^{0}(f)=f .
$$

(ii) For all $f \in L_{\gamma}^{2}\left(\mathbb{R}_{+}\right)$, we have

$$
\lim _{s \rightarrow 0^{+}} \mathscr{T}_{A}^{s}(f)=f, \quad \text { in } L_{\gamma}^{2}\left(\mathbb{R}_{+}\right)
$$

(iii) Let $1 \leq p<\infty$ and $s \geq t \geq 0$. Then, for all $\phi \in \mathscr{W}_{A}^{s, p}\left(\mathbb{R}_{+}\right)$, we have

$$
\left\|\mathscr{T}_{A}^{t}(\phi)\right\|_{\mathscr{W}_{A}^{s-t, p}\left(\mathbb{R}_{+}\right)}=\|\phi\|_{\mathscr{W}_{A}^{s, p}\left(\mathbb{R}_{+}\right)} .
$$


(iv) Let $s \geq t \geq 0$. Then, $\mathscr{W}_{A}^{t, 2}\left(\mathbb{R}_{+}\right) \subset \mathscr{W}_{A}^{s, 2}\left(\mathbb{R}_{+}\right)$. Moreover, for all $u \in \mathscr{W}_{A}^{t, 2}\left(\mathbb{R}_{+}\right)$, we have

$$
\|u\|_{\mathscr{W}_{A}^{s, 2}\left(\mathbb{R}_{+}\right)} \leq\|u\|_{\mathscr{W}_{A}^{t, 2}\left(\mathbb{R}_{+}\right)} .
$$

Proof. (i) For all $f \in \mathcal{S}_{*}^{\prime}(\mathbb{R})$, we have

$$
\begin{aligned}
\mathscr{T}_{A}^{s}\left(\mathscr{T}_{A}^{t}(f)\right) & =\mathscr{F}\left(e^{-s\left(\lambda^{2}+\rho^{2}\right)} e^{-t\left(\lambda^{2}+\rho^{2}\right)} \mathscr{F}^{-1}(f)\right) \\
& =\mathscr{F}\left(e^{-(t+s)\left(\lambda^{2}+\rho^{2}\right)} \mathscr{F}^{-1}(f)\right) \\
& =\mathscr{T}_{A}^{s+t}(f)
\end{aligned}
$$

and $\mathscr{T}_{A}^{0}(f)=\mathscr{F}\left(\mathscr{F}^{-1}(f)\right)=f$.

(ii) Clear.

(iii) Let $\phi \in \mathscr{W}_{A}^{s, p}\left(\mathbb{R}_{+}\right)$. By (i) and Definition 32, we obtain

$$
\begin{aligned}
& \left\|\mathscr{T}_{A}^{t}(\phi)\right\|_{\mathscr{W}_{A}^{s-t, p}\left(\mathbb{R}_{+}\right)} \\
& \quad=\left\|\mathscr{T}_{A}^{s-t}\left(\mathscr{T}_{A}^{t}(\phi)\right)\right\|_{L_{\gamma}^{p}\left(\mathbb{R}_{+}\right)}=\left\|\mathscr{T}_{A}^{s}(\phi)\right\|_{L_{\gamma}^{p}\left(\mathbb{R}_{+}\right)} \\
& \quad=\|\phi\|_{\mathscr{W}_{A}^{s, p}\left(\mathbb{R}_{+}\right)} .
\end{aligned}
$$

(iv) We deduce the result by using Proposition 4(ii).

Now, under a sufficient condition on $p$ and $q$, we shall prove that $\mathscr{T}_{A}^{s}$ is a bounded operator from $W_{A}^{t, p}\left(\mathbb{R}_{+}\right)$into $\mathscr{W}_{A}^{s, q}\left(\mathbb{R}_{+}\right)$.

Proposition 35. Let $s>0$ and $t \in \mathbb{R}$. Then, for all $q \geq 2$ and $p \geq q /(q-1)$, there exists a positive constant $C_{s, t}(p, q)$ such that for all $f \in W_{A}^{t, p}\left(\mathbb{R}_{+}\right)$, we have

$$
\|f\|_{\mathscr{W}_{A}^{s, q}\left(\mathbb{R}_{+}\right)} \leq C_{s, t}(p, q)\|f\|_{W_{A}^{t, p}\left(\mathbb{R}_{+}\right)} .
$$

Proof. According to Lemma 21 and Definition 32, we obtain the result by using Proposition 6 and applying Holder inequality.

4.2. Kernel Reproducing. Let $s \in \mathbb{R}$. The space $\mathscr{H}_{A}^{s}\left(\mathbb{R}_{+}\right)=$ $W_{A}^{s, 2}\left(\mathbb{R}_{+}\right)$provided with the inner product,

$$
\langle f, g\rangle_{\mathscr{H}_{A}^{s}\left(\mathbb{R}_{+}\right)}=\int_{\mathbb{R}_{+}}\left(1+x^{2}\right)^{2 s} \mathscr{J}(f)(x) \overline{\mathcal{F}(g)}(x) A(x) d x,
$$

and the norm $\|f\|_{\mathscr{H}_{A}^{s}\left(\mathbb{R}_{+}\right)}^{2}=\langle f, f\rangle_{\mathscr{H}_{A}^{s}\left(\mathbb{R}_{+}\right)}$, is a Hilbert space.

Proposition 36. For $s>3 / 4$ when $\rho>0$ and $s>(\alpha+1) / 2$ when $\rho=0$, the Hilbert space $\mathscr{H}_{A}^{s}\left(\mathbb{R}_{+}\right)$admits the following reproducing kernel:

$$
K_{s}(x, y)=\int_{\mathbb{R}_{+}} \frac{\varphi_{x}(\xi) \varphi_{y}(\xi) A(\xi) d \xi}{\left(1+\xi^{2}\right)^{2 s}} ;
$$

that is, (i) for all $y>0$, the function $x \mapsto K_{s}(x, y)$ belongs to $\mathscr{H}_{A}^{s}\left(\mathbb{R}_{+}\right)$.

(ii) The reproducing property: for all $f \in \mathscr{H}_{A}^{s}\left(\mathbb{R}_{+}\right)$and $y>$ 0 ,

$$
f(y)=\left\langle f, K_{s}(x, y)\right\rangle_{\mathscr{H}_{A}^{s}\left(\mathbb{R}_{+}\right)} .
$$

Proof. (i) It is clear from Lemma 21 and relations (9) and (10) that, for all $y>0$, the function

$$
\Theta_{y}: \xi \longmapsto \frac{\varphi_{y}(\xi)}{\left(1+\xi^{2}\right)^{2 s}}
$$

belongs to $L_{A}^{1}\left(\mathbb{R}_{+}\right) \cap L_{A}^{2}\left(\mathbb{R}_{+}\right)$when $s>3 / 4$ when $\rho>0$ and $s>(\alpha+1) / 2$ when $\rho=0$. Thus, the function $K_{s}(\cdot, y)$ is well defined and we can write

$$
K_{s}(x, y)=\mathscr{F}\left(\Theta_{y}\right)(x), \quad \forall x \in \mathbb{R}_{+} .
$$

Moreover, from Proposition 4, we can see that the function $K_{s}(\cdot, y)$ belongs to $L_{\gamma}^{2}\left(\mathbb{R}_{+}\right)$, and we have

$$
\mathscr{J}\left(K_{s}(\cdot, y)\right)(\xi)=\frac{\varphi_{y}(\xi)}{\left(1+\xi^{2}\right)^{2 s}}, \quad \forall \xi \in \mathbb{R}_{+} .
$$

Therefore, according to Lemma 21 and using relations (9) and (10), we deduce that

$$
\left\|K_{s}(\cdot, y)\right\|_{\mathscr{H}_{A}^{s}\left(\mathbb{R}_{+}\right)}^{2}<\infty .
$$

This proves that for all $y>0$, the function $K_{s}(\cdot, y)$ belongs to $\mathscr{H}_{A}^{s}\left(\mathbb{R}_{+}\right)$.

(ii) Let $f$ be in $\mathscr{H}_{A}^{s}\left(\mathbb{R}_{+}\right)$and $y>0$. Then by (121), we get

$$
\left\langle f, K_{s}(\cdot, y)\right\rangle_{\mathscr{H}_{A}^{s}\left(\mathbb{R}_{+}\right)}=\int_{\mathbb{R}_{+}} \mathscr{J}(f)(\xi) \varphi_{y}(\xi) A(\xi) d \xi,
$$

and from inversion formula, we obtain the reproducing property

$$
f(y)=\left\langle f, K_{s}(x, y)\right\rangle_{\mathscr{H}_{A}^{s}\left(\mathbb{R}_{+}\right)} .
$$

This completes the proof of the theorem.

Definition 37. For all positive real numbers $r, s$, and $t$, we define the Hilbert space $\mathscr{H}_{A}^{r, s, t}\left(\mathbb{R}_{+}\right)$as the subspace of $\mathscr{H}_{A}^{s}\left(\mathbb{R}_{+}\right)$with the inner product:

$$
\begin{array}{r}
\langle f, g\rangle_{\mathscr{H}_{A}^{r}, s, t\left(\mathbb{R}_{+}\right)}=r\langle f, h\rangle_{\mathscr{H}_{A}^{s}\left(\mathbb{R}_{+}\right)}+\left\langle\mathscr{T}_{A}^{t} f, \mathscr{T}_{A}^{t} g\right\rangle_{L_{\gamma}^{2}\left(\mathbb{R}_{+}\right)} \\
f, g \in \mathscr{H}_{A}^{s}\left(\mathbb{R}_{+}\right) .
\end{array}
$$

The norm associated to the inner product is defined by

$$
\|f\|_{\mathscr{H}_{A}^{\prime r s, t}\left(\mathbb{R}_{+}\right)}^{2}:=r\|f\|_{\mathscr{H}_{A}^{s}\left(\mathbb{R}_{+}\right)}^{2}+\|f\|_{\mathscr{W}_{A}^{t, 2}\left(\mathbb{R}_{+}\right)}^{2} .
$$

Proposition 38. Let $s>3 / 4$ when $\rho>0$ and $s>(\alpha+1) / 2$ when $\rho=0$. For all $r, t>0$, the Hilbert space $\mathscr{H}_{A}^{r, s, t}\left(\mathbb{R}_{+}\right)$admits the following reproducing kernel:

$$
\mathscr{K}_{r, s}^{t}(x, y)=\int_{\mathbb{R}_{+}} \frac{\varphi_{x}(\xi) \varphi_{y}(\xi)}{r\left(1+\xi^{2}\right)^{2 s}+e^{-2 t\left(\xi^{2}+\rho^{2}\right)}} A(\xi) d \xi .
$$


Proof. As in Proposition 36, we can deduce that for all $y>0$, there exists a function $x \mapsto \mathscr{K}_{r, s}^{t}(x, y)$ in $L_{\gamma}^{2}\left(\mathbb{R}_{+}\right)$such that we have

$$
\begin{gathered}
\mathscr{J}\left(\mathscr{K}_{r, s}^{t}(\cdot, y)\right)=\frac{\varphi_{y}}{r\left(1+\xi^{2}\right)^{2 s}+e^{-2 t\left(\xi^{2}+\rho^{2}\right)}}, \\
\left\|\mathscr{K}_{r, s}^{t}(\cdot, y)\right\|_{\mathscr{H}_{A}^{r, s}\left(\mathbb{R}_{+}\right)}^{2}<\infty .
\end{gathered}
$$

This proves that for all $y>0$ the function $\mathscr{K}_{r, s}^{t}(\cdot, y)$ belongs to $\mathscr{H}_{A}^{r, s, t}\left(\mathbb{R}_{+}\right)$.

On the other hand, for $f$ in $\mathscr{H}_{A}^{r, s, t}\left(\mathbb{R}_{+}\right)$and $y>0$, we have

$$
\left\langle f, \mathscr{K}_{r, s}^{t}(\cdot, y)\right\rangle_{\mathscr{H}_{A}^{r, s, t}\left(\mathbb{R}_{+}\right)}=r I_{1}+I_{2},
$$

where

$$
\begin{gathered}
I_{1}=\left\langle f, \mathscr{K}_{r, s}^{t}(\cdot, y)\right\rangle_{\mathscr{H}_{A}^{s}\left(\mathbb{R}_{+}\right)}, \\
I_{2}=\left\langle\mathscr{T}_{A}^{t} f, \mathscr{T}_{A}^{t}\left(\mathscr{K}_{r, s}^{t}(\cdot, y)\right)\right\rangle_{L_{\gamma}^{2}\left(\mathbb{R}_{+}\right)} .
\end{gathered}
$$

But from (116) and (128), we have

$$
I_{1}=\int_{\mathbb{R}_{+}} \frac{\left(1+\xi^{2}\right)^{2 s} \mathscr{J}(f)(\xi) \varphi_{y}(\xi)}{r\left(1+\xi^{2}\right)^{2 s}+e^{-2 t\left(\xi^{2}+\rho^{2}\right)}} A(\xi) d \xi .
$$

and from (128), it follows, by using Parseval formula for the transform $\mathscr{F}$, that

$$
\begin{aligned}
I_{2}= & \int_{\mathbb{R}_{+}} \mathscr{F}\left(e^{-t\left(x^{2}+\rho^{2}\right)} \mathscr{J}(f)\right)(\lambda) \mathscr{F} \\
& \times\left(e^{-t\left(x^{2}+\rho^{2}\right)} \mathscr{J}\left(\mathscr{K}_{r, s}^{t}(\cdot, y)\right)\right)(\lambda) d \gamma(\lambda) \\
= & \int_{\mathbb{R}_{+}} \frac{e^{-2 t\left(\xi^{2}+\rho^{2}\right)} \mathscr{J}(f)(\xi) \varphi_{y}(\xi)}{r\left(1+\xi^{2}\right)^{2 s}+e^{-2 t\left(\xi^{2}+\rho^{2}\right)}} A(\xi) d \xi .
\end{aligned}
$$

Thus, by virtue of (129), and combining (131) and (132), we deduce that

$$
\left\langle f, \mathscr{K}_{r, s}^{t}(\cdot, y)\right\rangle_{\mathscr{H}_{A}^{r, s, t}\left(\mathbb{R}_{+}\right)}=f(y), \quad \text { a.e. }
$$

4.3. Extremal Function for Generalized Weierstrass Transform. In this subsection, we show the existence and unicity of the extremal function related to the generalized Weierstrass transform $\mathscr{T}_{A}^{t}$. We start with the following fundamental theorem (cf. [20]).

Theorem 39. Let $H_{K}$ be a Hilbert space admitting the reproducing kernel $K(p, q)$ on a set $E$ and let $H$ be a Hilbert space. Let $L: H_{K} \rightarrow H$ be a bounded linear operator on $H_{K}$ into $H$. For $r>0$, we introduce the inner product in $H_{K}$ and we call it $H_{K_{r}}$ as

$$
\left\langle f_{1}, f_{2}\right\rangle_{H_{K_{r}}}=r\left\langle f_{1}, f_{2}\right\rangle_{H_{K}}+\left\langle L f_{1}, L f_{2}\right\rangle_{H}
$$

Then, (i) $H_{K_{r}}$ is a Hilbert space with the reproducing kernel $K_{r}(p, q)$ on $E$ and satisfying the equation

$$
K(\cdot, q)=\left(r I+L^{*} L\right) K_{r}(\cdot, q),
$$

where $L^{*}$ is the adjoint operator of $L: H_{K} \rightarrow H$.

(ii) For any $r>0$ and for any $h$ in $H$, the infinitum

$$
\inf _{f \in H_{K}}\left\{r\|f\|_{H_{K}}^{2}+\|L f-h\|_{H}^{2}\right\}
$$

is attained by a unique function $f_{r, h}^{*}$ in $H_{K}$ and this extremal function is given by

$$
f_{r, h}^{*}(p)=\left\langle h, L K_{r}(\cdot, p)\right\rangle_{H} \cdot
$$

We can now state the main result of this paragraph.

Theorem 40. Let $s>3 / 4$ when $\rho>0$ and $s>(\alpha+1) / 2$ when $\rho=0$.

(i) For any $g \in L_{\gamma}^{2}\left(\mathbb{R}_{+}\right)$and for any $r>0$, the best approximate function $f_{r, g}^{*}$ in the sense

$$
\begin{array}{r}
\inf _{f \in \mathscr{H}_{A}^{s}\left(\mathbb{R}_{+}\right)}\left\{r\|f\|_{\mathscr{H}_{A}^{s}\left(\mathbb{R}_{+}\right)}^{2}+\left\|g-\mathscr{T}_{A}^{t} f\right\|_{L_{\gamma}^{2}\left(\mathbb{R}_{+}\right)}^{2}\right\} \\
=r\left\|f_{r, g}^{*}\right\|_{\mathscr{H}_{A}^{s}\left(\mathbb{R}_{+}\right)}^{2}+\left\|g-\mathscr{T}_{A}^{t} f_{r, g}^{*}\right\|_{L_{\gamma}^{2}\left(\mathbb{R}_{+}\right)}^{2}
\end{array}
$$

exists uniquely and $f_{r, g}^{*}$ is represented by

$$
f_{r, g}^{*}(y)=\int_{\mathbb{R}_{+}} g(x) Q_{r}(x, y) d \gamma(x),
$$

where

$Q_{r}(x, y)=\int_{\mathbb{R}_{+}} \frac{e^{-2 t\left(\xi^{2}+\rho^{2}\right)} \varphi_{x}(\xi) \varphi_{y}(\xi)}{r\left(1+\xi^{2}\right)^{2 s}+e^{-2 t\left(\xi^{2}+\rho^{2}\right)}} A(\xi) d \xi$.

(ii) Let $f \in \mathscr{H}_{A}^{s}\left(\mathbb{R}_{+}\right)$. Then, If we take $g=\mathscr{T}_{A}^{t} f$, we have

$$
f_{r, g}^{*} \longrightarrow f \text { as } r \longrightarrow 0^{+} \text {, uniformly. }
$$

(iii) Let $\delta>0$ and let $g$ and $g_{\delta}$ satisfy $\left\|g-g_{\delta}\right\|_{L_{\gamma}^{2}\left(\mathbb{R}_{+}\right)} \leq \delta$. Then

$$
\left\|f_{r, g}^{*}-f_{r, g_{\delta}}^{*}\right\|_{\mathscr{H}_{A}^{s}\left(\mathbb{R}_{+}\right)} \leq \frac{\delta}{\sqrt{r}} .
$$

Proof. (i) By Proposition 38 and Theorem 39(ii), the infinitum given by (138) is attained by a unique function $f_{r, g}^{*}$, and the extremal function $f_{r, g}^{*}$ is represented by

$$
f_{r, g}^{*}(y)=\left\langle g, \mathscr{T}_{A}^{t}\left(\mathscr{K}_{g, r}^{t}(\cdot, y)\right)\right\rangle_{L_{\gamma}^{2}\left(\mathbb{R}_{+}\right)}, \quad y>0,
$$

where $\mathscr{K}_{g, r}^{t}$ is the kernel given by Proposition 38. Hence, by (128), we obtain the expression (140) of $Q_{r}(x, y)$. 
(ii) From Proposition 35, the function $g$ belongs to $L_{\gamma}^{2}\left(\mathbb{R}_{+}\right)$. According to Lemma 21 and relations (9) and (10), it can be observed, using Cauchy-Schwartz inequality, that for all $y>0$ the function

$$
\xi \longmapsto \mathscr{J}(f)(\xi) \varphi_{y}(\xi)
$$

belongs to $L_{A}^{1}\left(\mathbb{R}_{+}\right)$, which implies, from inversion formula for the transform $\mathcal{F}$, that

$$
f(y)=\int_{\mathbb{R}_{+}} \mathscr{J}(f)(\xi) \varphi_{y}(\xi) A(\xi) d \xi .
$$

Therefore, by (132), it follows that

$$
\left(f_{r, g}^{*}-f\right)(y)=\int_{\mathbb{R}_{+}} \frac{-r\left(1+\xi^{2}\right)^{2 s} \mathscr{J}(f)(\xi) \varphi_{y}(\xi)}{r\left(1+\xi^{2}\right)^{2 s}+e^{-2 t\left(\xi^{2}+\rho^{2}\right)}} A(\xi) d \xi .
$$

Hence, by dominated convergence theorem we deduce the result.

(iii) It is clear, from (140), that

$$
Q_{r}(x, y)=\mathscr{F}\left(\frac{e^{-2 t\left(\xi^{2}+\rho^{2}\right)} \varphi_{x}(\xi)}{r\left(1+\xi^{2}\right)^{2 s}+e^{-2 t\left(\xi^{2}+\rho^{2}\right)}}\right)(y),
$$

then, using Parseval formula for the Fourier transform $\mathscr{F}$, it follows, by (139), that

$$
f_{r, g}^{*}(x)=\int_{\mathbb{R}_{+}} \frac{e^{-2 t\left(\xi^{2}+\rho^{2}\right)} \mathscr{J}(g)(\xi) \varphi_{x}(\xi)}{r\left(1+\xi^{2}\right)^{2 s}+e^{-2 t\left(\xi^{2}+\rho^{2}\right)}} A(\xi) d \xi
$$

and so

$$
\mathscr{J}\left(f_{r, g}^{*}\right)(\xi)=\frac{e^{-2 t\left(\xi^{2}+\rho^{2}\right)} \mathscr{J}(g)(\xi)}{r\left(1+\xi^{2}\right)^{2 s}+e^{-2 t\left(\xi^{2}+\rho^{2}\right)}}
$$

Hence,

$$
\mathscr{J}\left(f_{r, g}^{*}-f_{r, g_{\delta}}^{*}\right)(\xi)=\frac{e^{-2 t\left(\xi^{2}+\rho^{2}\right)} \mathscr{J}\left(g-g_{\delta}\right)(\xi)}{r\left(1+\xi^{2}\right)^{2 s}+e^{-2 t\left(\xi^{2}+\rho^{2}\right)}} .
$$

Using the inequality $(x+y)^{2} \geq 4 x y$, we obtain

$$
\left(1+\xi^{2}\right)^{2 s}\left|\mathscr{J}\left(f_{r, g}^{*}-f_{r, g_{\delta}}^{*}\right)(\xi)\right|^{2} \leq \frac{1}{4 r}\left|\mathscr{J}\left(g-g_{\delta}\right)(\xi)\right|^{2}
$$

Thus, and from Proposition 4(ii), we obtain

$$
\begin{aligned}
\left\|f_{r, g}^{*}-f_{r, g_{\delta}}^{*}\right\|_{\mathscr{H}_{A}^{s}\left(\mathbb{R}_{+}\right)}^{2} & \leq \frac{1}{4 r}\left\|\mathscr{J}\left(g-g_{\delta}\right)\right\|_{L_{A}^{2}\left(\mathbb{R}_{+}\right)}^{2} \\
& =\frac{1}{4 r}\left\|g-g_{\delta}\right\|_{L_{\gamma}^{2}\left(\mathbb{R}_{+}\right)}^{2},
\end{aligned}
$$

which gives the desired result.

\section{Conflict of Interests}

The authors declare that there is no conflict of interests regarding the publication of this paper.

\section{References}

[1] W. R. Bloom and H. Heyer, Harmonic Analysis of Probability Measures on Hypergroups, vol. 20 of de Gruyter Studies in Mathematics, Walter de Gruyter, Berlin, Germany, 1995.

[2] M. Assal and M. M. Nessibi, "Bessel-Sobolev type spaces," Mathematica Balkanica, vol. 18, no. 3-4, pp. 227-234, 2004.

[3] N. Ben Salem and A. Dachraoui, "Sobolev type spaces associated with the Jacobi operator," Integral Transforms and Special Functions, vol. 7, no. 1, 1998.

[4] W. R. Bloom and Z. Xu, "Fourier multipliers for local Hardy spaces on Chébli-Trimèche hypergroups," Canadian Journal of Mathematics, vol. 50, no. 5, pp. 897-928, 1998.

[5] M. Dziri, M. Jelassi, and L. T. Rachdi, "Spaces of $D L_{p}$ type and a convolution product associated with a singular second order differential operator," Journal of Concrete and Applicable Mathematics, vol. 10, no. 3-4, pp. 207-232, 2012.

[6] K. Trimèche, "Inversion of the Lions transmutation operators using generalized wavelets," Applied and Computational Harmonic Analysis, vol. 4, no. 1, pp. 97-112, 1997.

[7] K. Trimèche, “Transformation intégrale de Weyl et théorème de Paley-Wiener associés à un opérateur différentiel singulier sur $(0, \infty)$," Journal de Mathématiques Pures et Appliquées, vol. 60, no. 1, pp. 51-98, 1981.

[8] Z. Xu, Harmonic Analysis on Chébli-Trimèche Hypergroups [Ph.D. thesis], Murdoch University, Perth, Australia, 1994.

[9] H. Chebli, "Théorème de Paley-Wiener associé à un opérateur


Pures et Appliquées, vol. 58, no. 1, pp. 1-19, 1979.

[10] H. Chebli, "Opérateurs de translation généralisée et semigroupes de convolution," in Théorie du Potentiel et Analyse Harmonique, vol. 404 of Lecture Notes in Mathematics, pp. 3559, Springer, Berlin, Germany, 1974.

[11] E. C. Titchmarsh, Introduction to the Theory of Fourier Integrals, Clarendon Press, Oxford, UK, 1937.

[12] G. B. Folland, Real Analysis Modern Techniques and Their Applications, Pure and Applied Mathematics (New York), John Wiley \& Sons, A Wiley-Interscience Publication, New York, NY, USA, 1984.

[13] W. Rudin, Analyse Fonctionnelle, Ediscience International, Paris, France, 1995.

[14] H. Brezis, Analyse Fonctionnelle, Masson, Paris, France, 3rd edition, 1983.

[15] L. Bouattour and K. Trimèche, "Beurling-Hörmander's theorem for the Chébli-Trimèche transform," Global Journal of Pure and Applied Mathematics, vol. 1, no. 3, pp. 342-357, 2005.

[16] S. Omri and L. T. Rachdi, "Weierstrass transform associated with the Hankel operator," Bulletin of Mathematical Analysis and Applications, vol. 1, no. 2, pp. 1-16, 2009.

[17] T. Matsuura, S. Saitoh, and D. D. Trong, "Approximate and analytical inversion formulas in heat conduction on multidimensional spaces," Journal of Inverse and Ill-Posed Problems, vol. 13, no. 3-6, pp. 479-493, 2005.

[18] S. Saitoh, "Approximate real inversion formulas of the Gaussian convolution," Applicable Analysis, vol. 83, no. 7, pp. 727-733, 2004 . 
[19] S. Saitoh, "The Weierstrass transform and an isometry in the heat equation," Applicable Analysis, vol. 16, no. 1, pp. 1-6, 1983.

[20] S. Saitoh, Theory of Reproducing Kernels and its Applications, vol. 189 of Pitman Research Notes in Mathematics Series, Longman Scientific \& Technical, Harlow, UK, 1988. 


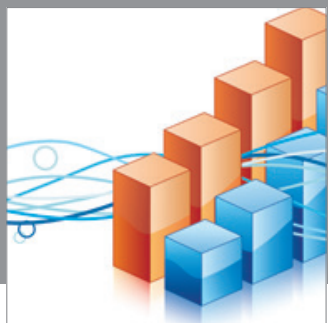

Advances in

Operations Research

mansans

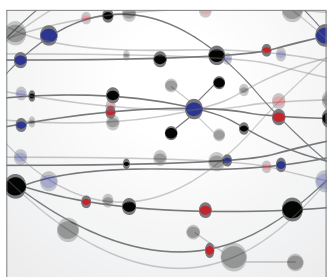

The Scientific World Journal
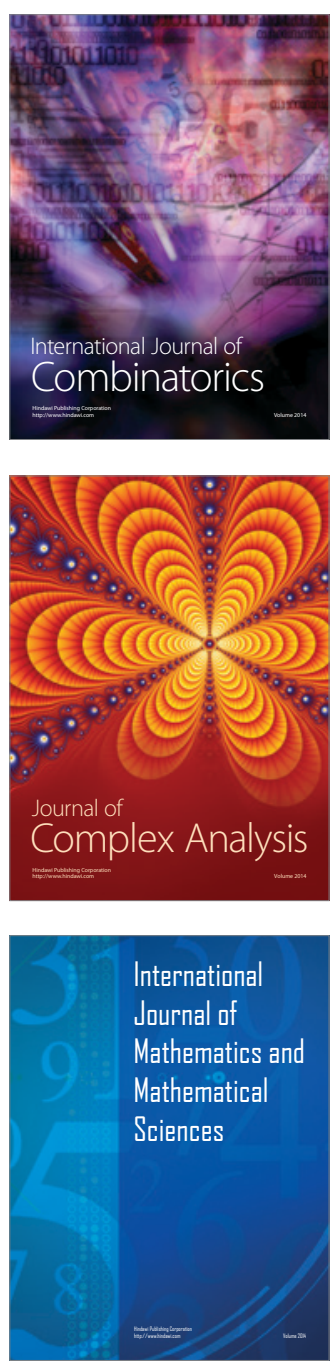
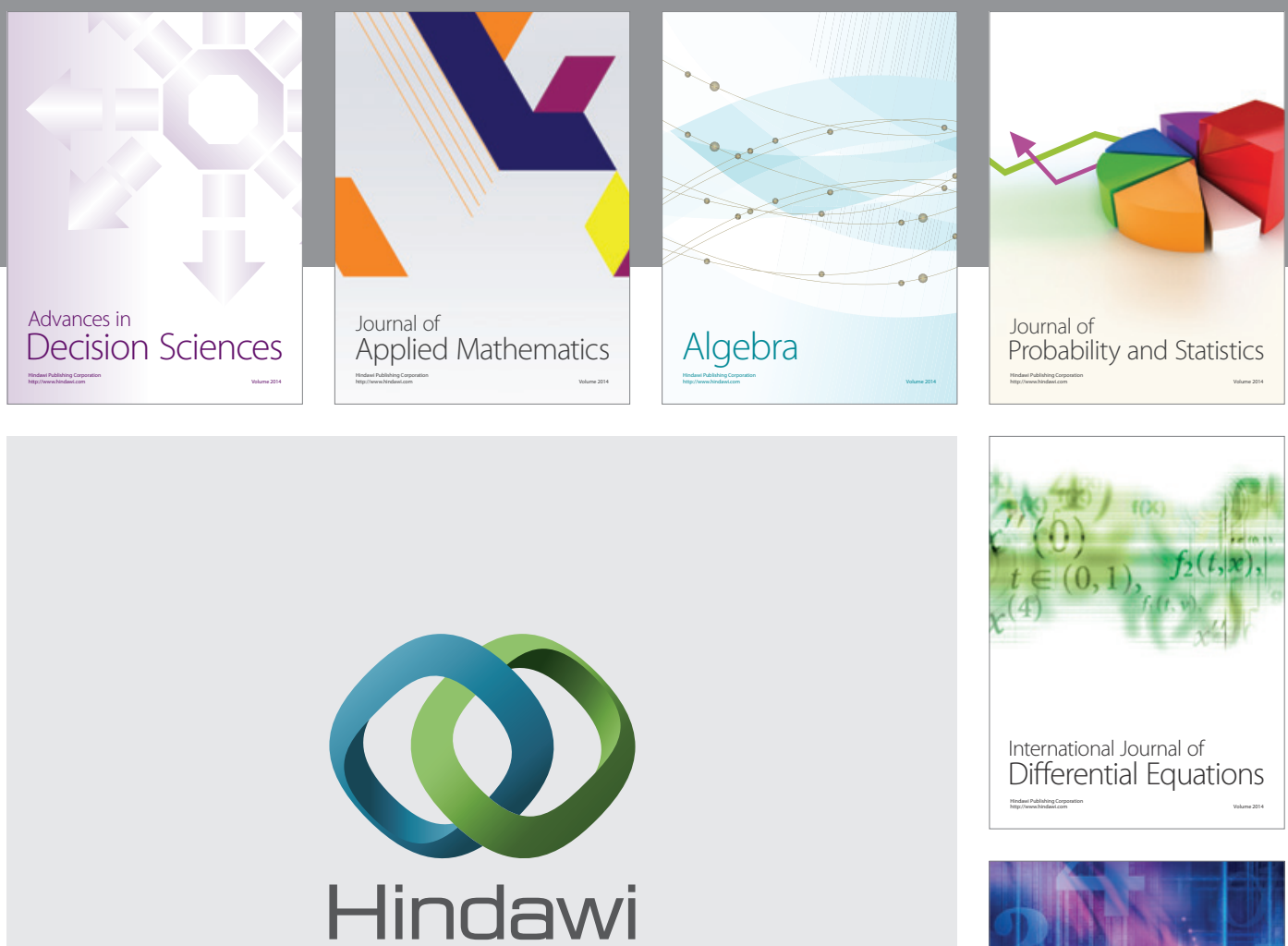

Submit your manuscripts at http://www.hindawi.com
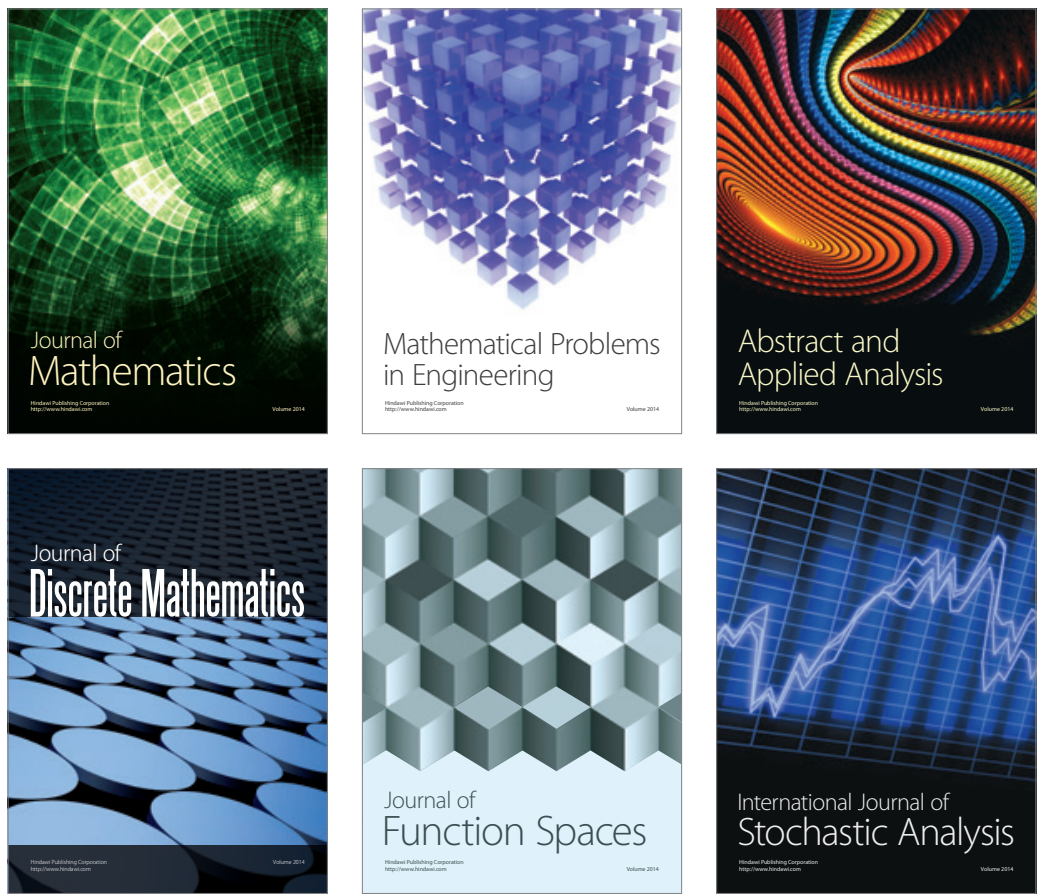

Journal of

Function Spaces

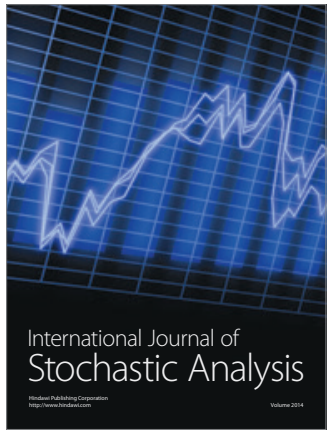

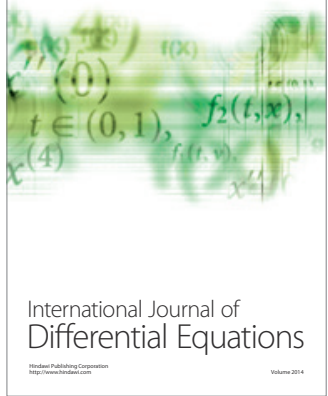
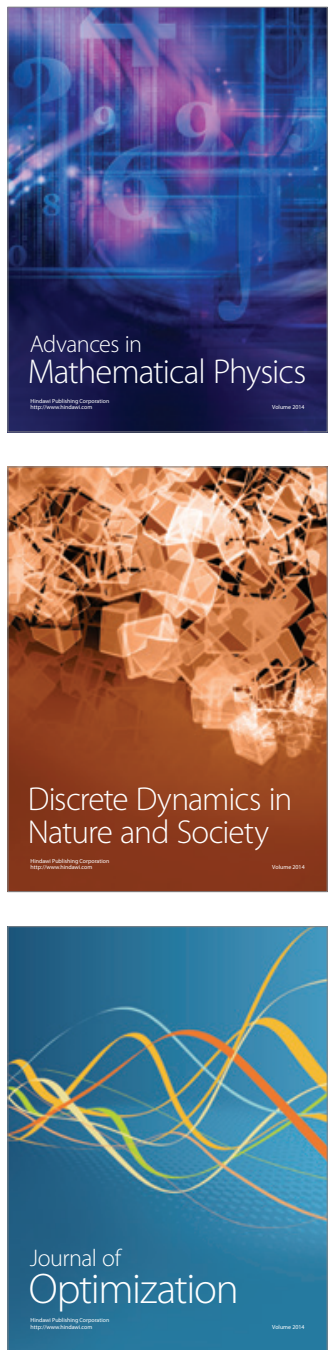